\title{
THE DIRAC EQUATION IN TWO DIMENSIONS: DISPERSIVE ESTIMATES AND CLASSIFICATION OF THRESHOLD OBSTRUCTIONS
}

\author{
M. BURAK ERDOĞGN AND WILLIAM R. GREEN
}

\begin{abstract}
We investigate dispersive estimates for the two dimensional Dirac equation with a potential. In particular, we show that the Dirac evolution satisfies a $t^{-1}$ decay rate as an operator from the Hardy space $H^{1}$ to $B M O$, the space of functions of bounded mean oscillation. This estimate, along with the $L^{2}$ conservation law allows one to deduce a family of Strichartz estimates. We classify the structure of threshold obstructions as being composed of s-wave resonances, p-wave resonances and eigenfunctions. We show that, as in the case of the Schrödinger evolution, the presence of a threshold s-wave resonance does not destroy the $t^{-1}$ decay rate. As a consequence of our analysis we obtain a limiting absorption principle in the neighborhood of the threshold, and show that there are only finitely many eigenvalues in the spectral gap.
\end{abstract}

\section{INTRODUCTION}

We consider the linear Dirac equation with a potential:

$$
i \partial_{t} \psi(x, t)=\left(D_{m}+V(x)\right) \psi(x, t), \quad \psi(x, 0)=\psi_{0}(x) .
$$

Here the spatial variable $x \in \mathbb{R}^{n}$, and $\psi(x, t) \in \mathbb{C}^{2^{n-1}}$. The free Dirac operator $D_{m}$ is defined by

$$
D_{m}=-i \alpha \cdot \nabla+m \beta=-i \sum_{k=1}^{n} \alpha_{k} \partial_{k}+m \beta
$$

where $m>0$ is a constant, and the $n \times n$ Hermitian matrices $\alpha_{0}:=\beta$ and $\alpha_{j}$ satisfy

$$
\alpha_{j} \alpha_{k}+\alpha_{k} \alpha_{j}=2 \delta_{j k} \mathbb{1}_{\mathbb{C}^{2 n-1}}, \quad j, k \in\{0,1,2, \ldots, n\}
$$

For concreteness, in two dimensions we use

$$
\beta=\left(\begin{array}{cc}
1 & 0 \\
0 & -1
\end{array}\right), \quad \alpha_{1}=\left(\begin{array}{cc}
0 & 1 \\
1 & 0
\end{array}\right), \quad \alpha_{2}=\left(\begin{array}{cc}
0 & -i \\
i & 0
\end{array}\right) .
$$

Dirac arrived at these equations to describe the evolution of an electron moving at relativistic speeds, thus the Dirac equation is a way to connect the physical theories of quantum mechanics and relativity, see, e.g., [42]. The Dirac equation can be derived by applying quantum-mechanical notions of energy $E=i \hbar \partial_{t}$ and momentum $p=-i \hbar \nabla$ to the relativistic relationship between

The first author was partially supported by the NSF grant DMS-1501041. 
energy, momentum and mass, $E=\sqrt{c^{2} p^{2}+m^{2} c^{4}}$. One arrives at the square root of a KleinGordon equation,

$$
i \hbar \partial_{t} \psi(x, t)=\sqrt{-c^{2} \hbar^{2} \Delta+m^{2} c^{4}} \psi(x, t)
$$

Here $\hbar$ is Planck's constant and $c$ is the speed of light. In our mathematical analysis, we rescale all constants to be one. Dirac's linearization of the above equation led to the free Dirac equation, a system of coupled hyperbolic equations, (11) with $V=0$. Dirac's linearization allows one to account for the spin of quantum particles, as well as providing a way to incorporate external electro-magnetic fields in a manner compatible with the relativistic theory where the Klein-Gordon model cannot. Further details can be found in [42].

The following identity 1 which follows from (3),

$$
\left(D_{m}-\lambda \mathbb{1}\right)\left(D_{m}+\lambda \mathbb{1}\right)=(-i \alpha \cdot \nabla+m \beta-\lambda \mathbb{1})(-i \alpha \cdot \nabla+m \beta+\lambda \mathbb{1})=\left(-\Delta+m^{2}-\lambda^{2}\right)
$$

allows us to formally define the free Dirac resolvent operator $\mathcal{R}_{0}(\lambda)=\left(D_{m}-\lambda\right)^{-1}$ in terms of the free resolvent $R_{0}(\lambda)=(-\Delta-\lambda)^{-1}$ of the Schrödinger operator for $\lambda$ in the resolvent set:

$$
\mathcal{R}_{0}(\lambda)=\left(D_{m}+\lambda\right) R_{0}\left(\lambda^{2}-m^{2}\right) .
$$

We note that

$$
\sigma\left(D_{m}\right)=\sigma_{e s s}\left(D_{m}\right)=(-\infty,-m] \cup[m, \infty)
$$

and for suitable potential functions $V$, one has $\sigma_{e s s}(H)=\sigma_{e s s}\left(D_{m}\right)$ with $H=D_{m}+V$. This is satisfied for large classes of potentials, for examples if $V(x) \rightarrow 0$ as $|x| \rightarrow \infty$, see [42, Theorem 4.7], or [4]. For the class of potentials we consider in this paper, Georgescu and Mantoiu [29, Theorem 1.4] proved that there is no singular continuous spectrum of $H$, also see [46]. Furthermore, the set of eigenvalues is a discrete subset of $\mathbb{R} \backslash\{m,-m\}$, and each eigenvalue is of finite multiplicity, see [29] and [16]. It is possible that eigenvalues accumulate at $\pm m$ even for bounded and decaying potentials, see [42]. However, the resolvent expansions we obtain in Section 4 rule that out, see Remark 4.7. In particular there are finitely many eigenvalues in the spectral gap $(-m, m)$; also see [38], 36], 37], and [15]. In three dimensions, for suitably decaying potentials, it is known that there are no embedded eigenvalues in the essential spectrum, except possibly at the thresholds $\lambda= \pm m$, 43]; also see also [40, 8, 45, 29]. Although the method in [43] appears to be applicable in general dimensions and for more general operators, this has not been pursued anywhere. The method of Roze in [40] is based on squaring the equation and using analogous results for Schrodinger type operators. This is applicable in two dimensions, however it only applies for potentials of the form $q(x) I$.

For our analysis, we make the following assumptions on the potential $V$ and the spectrum of $H=D_{m}+V$.

\footnotetext{
${ }^{1}$ Here and throughout the paper, scalar operators such as $-\Delta+m^{2}-\lambda^{2}$ are understood as $\left(-\Delta+m^{2}-\lambda^{2}\right) \mathbb{1}_{\mathbb{C}^{2}}{ }^{n-1}$.
} 
Assumption 1.1. i) The matrix-valued potential function $V(x)$ is self-adjoint and its entries satisfy the decay estimate $\left|V_{i j}(x)\right| \lesssim\langle x\rangle^{-\gamma}$ for some $\gamma>3$.

ii) There are no eigenvalues in $(-\infty,-m) \cup(m, \infty)$.

iii) A limiting absorption principle for the perturbed resolvent operator of the form:

$$
\sup _{|\lambda|>\lambda_{0}}\left\|\partial_{\lambda}^{k} \mathcal{R}_{V}^{ \pm}(\lambda)\right\|_{L^{2, \sigma} \rightarrow L^{2,-\sigma}} \lesssim 1, \quad \sigma>\frac{1}{2}+k, \quad k=0,1,
$$

holds for any $\lambda_{0}>m$.

We note that the second and third assumptions are used only in the high energy analysis of the operator. The third assumption above requires some discussion. Note that in contrast with the Schrödinger resolvent, the resolvent for the free Dirac operator does not decay as $\lambda \rightarrow \infty$. Therefore, Agmon's bootstrapping argument 2] produces uniform bounds in $\lambda$ only for compact subsets of the purely absolutely continuous spectrum, see e.g. [46], [29]. In particular, under the first two assumptions, the results of [29] imply that

$$
\sup _{\lambda_{1}>|\lambda|>\lambda_{0}}\left\|\partial_{\lambda}^{k} \mathcal{R}_{V}^{ \pm}(\lambda)\right\|_{L^{2, \sigma} \rightarrow L^{2,-\sigma}} \lesssim 1, \quad \sigma>\frac{1}{2}+k, \quad k=0,1,
$$

for any $\lambda_{1}>\lambda_{0}>m$, with a bound depending on both $\lambda_{1}$ and $\lambda_{0}$. This situation is quite similar to the case of magnetic Schrödinger equation, and it is likely that one can obtain (7) under the first two assumptions using the method in [21]. This will be pursued elsewhere.

The limiting absorption principle is much better studied in the three dimensional case, see for example [10, 19, 12]. The results of D'Ancona and Fanelli, [19], requires the potential to be small. Boussaid and Golénia, 12, established a limiting absorption principle near the thresholds $\lambda= \pm m$. Finally, Georgescu and Mantoiu [29] obtained a limiting absorption principle in general dimensions but it is only uniform on compact subsets of the purely absolutely continuous spectrum.

As our time decay analysis requires only the decay assumption but no smoothness or smallness in any particular norm, we chose to leave this as an overarching assumption. In particular, we need only use this assumption in our high energy analysis in Section 6. A viable limiting absorption principle of this form may be attained for a non-trivial class of potentials, following the work of Boussaid [10] in three dimensions provided the potential is $C^{\infty}$ and satisfies the decay estimates $\left|\partial_{x}^{k} V(x)\right| \lesssim\langle x\rangle^{-5-k-}$. This approach makes use of the minimal escape velocity estimates of Hunziker, Sigal and Soffer, [33], to establish time decay on polynomially weighted $L^{2}$ spaces. Then, one can use iterated resolvent identities to establish (7), see [36].

To state our main result we introduce some notation. Throughout the paper $a-:=a-\epsilon$ for an arbitrarily small, but fixed, $\epsilon>0$. Similarly, $a+:=a+\epsilon$. Let $P_{a c}$ be the spectral projection onto the absolutely continuous spectrum. For the definition of the threshold regularity and resonances see Definition 4.3 below. 
Theorem 1.2. Suppose Assumption 1.1 holds, and the threshold energies, $\pm m$, are regular or there are resonances of the first kind at $\pm m$. Then, for any $0 \leq \alpha \leq 1$, we have the dispersive bounds for the Dirac evolution 2

$$
\left\|e^{-i t H} P_{a c}(H)\langle H\rangle^{-2-\frac{3}{2} \alpha-} f\right\|_{B M O} \lesssim \frac{1}{\langle t\rangle^{\alpha}}\|f\|_{H^{1}} .
$$

The free Dirac evolution has threshold s-wave resonances, thus Theorem 1.2 holds under the natural conditions on the edge of the spectrum.

As usual, we study the dispersive bounds on the evolution by expressing $e^{-i t H} P_{a c}(H)$ via the Stone's formula:

$$
e^{-i t H} P_{a c}(H) f(x)=\frac{1}{2 \pi} \int_{\sigma_{e s s}(H)} e^{-i t \lambda}\left[\mathcal{R}_{V}^{+}-\mathcal{R}_{V}^{-}\right](\lambda) f(x) d \lambda .
$$

Due to the differing behavior of the resolvents $\mathcal{R}_{V}^{ \pm}(\lambda)$ in a neighborhood of the threshold and away from the threshold on the positive half of the spectrum, our analysis proceeds in two cases. We first consider the low-energy contribution, when $\lambda$ is in a sufficiently small neighborhood of the threshold $\lambda=m$. A similar analysis can be done on the negative portion of the spectrum.

Theorem 1.3. Under Assumption 1.1 part i), with $\chi$ a smooth cut-off to a sufficiently small neighborhood of the threshold energy $\lambda=m$. If $\lambda=m$ is regular or if there is a resonance of the first kind, then the following dispersive bound holds.

$$
\left\|e^{-i t H} P_{a c}(H) \chi(H) f\right\|_{B M O} \lesssim \frac{1}{\langle t\rangle}\|f\|_{H^{1}} .
$$

When $\lambda$ is away from the threshold, we consider the evolution concentrated on dyadic frequencies to prove the following.

Theorem 1.4. Under Assumption 1.1, with $\chi_{j}$ a smooth cut-off to $\lambda \approx 2^{j}, j \geq 0$, we have the bound

$$
\left\|e^{-i t H} P_{a c}(H) \chi_{j}(H) f\right\|_{B M O} \lesssim \min \left(2^{2 j}, 2^{\frac{7}{2} j}|t|^{-1}\right)\|f\|_{H^{1}}
$$

Therefore, we obtain

$$
\left\|e^{-i t H}\langle H\rangle^{-\frac{7}{2}-} P_{a c}(H) f\right\|_{B M O} \lesssim|t|^{-1}\|f\|_{H^{1}} .
$$

Interpolating this bound with the $L^{2}$ conservation (see, e.g. [7, 32]) one obtains the $L^{p} \rightarrow L^{p^{\prime}}$ bound:

$$
\left\|e^{-i t H}\langle H\rangle^{\frac{7}{2}-\frac{7}{p}-} P_{a c}(H) f\right\|_{L^{p^{\prime}}} \lesssim|t|^{1-\frac{2}{p}}\|f\|_{L^{p}}, \quad 1<p \leq 2 .
$$

As in the seminal work of Ginibre and Velo [30], this yields the following Strichartz estimates:

\footnotetext{
${ }^{2}$ Thoroughout the paper $\|\cdot\|_{B M O}$ denotes $\|\cdot\|_{B M O \times B M O\left(\mathbb{R}^{2}\right)}$. We similarly use the notation $\|\cdot\|_{H^{1}}$ and $\|\cdot\|_{L^{p}}$. See [28] for the definition of the spaces $H^{1}$ and $B M O$.
} 
Corollary 1.5. Under the conditions of Theorem 1.2, we have

$$
\left\|e^{-i t H}\langle H\rangle^{\frac{7}{2 r}-\frac{7}{4}-} P_{a c}(H) f\right\|_{L_{t}^{q} L_{x}^{r}} \lesssim\|f\|_{L^{2}}, \frac{1}{q}+\frac{1}{r}=\frac{1}{2}, \quad 2 \leq r<\infty .
$$

Since the time-decay we obtain is the same as that for the Schrödinger equation in two dimensions, the range of admissible exponents for the Dirac evolution mirrors that of Schrödinger evolution.

The mathematical analysis of Dirac operators is less well-studied than the related Schrödinger, wave and Klein-Gordon equations. The paper [19] by D'Ancona and Fanelli seems to be the first to analyze the time-decay for a perturbed Dirac evolution in a pointwise sense. This analysis in the three-dimensional case considered the massless Dirac equation $(m=0)$ and related wave equations with small electromagnetic potentials. Dispersive and Strichartz estimates for the free Dirac equation in three dimension 3 were proven by Escobedo and Vega, [27], to analyze a semi-linear Dirac equation. Boussaid, [10] proved dispersive estimates in Besov spaces and weighted $L^{2}$ spaces for the three-dimensional Dirac equation with mass $m>0$, with an aim towards studying the stable manifold around 'particle-like solutions' for a class of non-linear Dirac equations.

Further study of the Dirac operator in the sense of smoothing and Strichartz estimates has been performed by a variety of authors, see for example [11, 13, 14]. In the two-dimensional case, the evolution on weighted $L^{2}$ spaces was studied in [36], which had roots in the work of Murata, [38]. Frequency-localized endpoint Strichartz estimates for the free Dirac equation are obtained in two spatial dimensions in [6], which are used to study the cubic non-linear Dirac equation. Dispersive estimates for one-dimensional Dirac equation was considered in [17].

Our approach relies on a detailed analysis of the Dirac resolvent operators. We follow the strategy employed to analyze the two-dimensional Schrödinger equation set out in [41] and in our earlier work [23, 24, 25], also see [44]. We note that extending these results is non-trivial even for the wave equation, see [31, 5]. In contrast to the Schrödinger and wave equation, we present our estimates from $H^{1}$ to $B M O$ instead of as operators from $L^{1} \rightarrow L^{\infty}$. The use of such spaces are necessitated by technical issues which we discuss in Section 5 , however they still serve as interpolation spaces in the same way as $L^{1}$ and $L^{\infty}$. Dispersive estimates in the setting of $H^{1}$ and $B M O$ spaces were established in [18, 5].

In addition to proving time decay estimates for the Dirac evolution, we provide a full classification of the obstructions that can occur at the threshold of the essential spectrum at $\lambda= \pm m$. In two dimensions, there is a rich structure of resonances and eigenfunctions that can occur, which we classify. This classification is inspired by the previous work on Schrödinger operators

\footnotetext{
${ }^{3}$ During the review period for this article, the authors and Toprak studied the analagous dispersive estimates for three dimensional Dirac equations with threshold obstructions, 26].
} 
[35, 9, 23]. For the classification of threshold obstructions in three dimensions and their effect on the time decay of the Dirac evolution see [26].

The paper is organized as follows. We first develop expansions for the free Dirac resolvent around the threshold energy $\lambda=m$ in Section 2. These bounds allow us to prove the natural time decay bounds for the free Dirac evolution as an operator between Besov spaces in Section 3 as well as to establish expansions for the perturbed resolvent near the threshold in Section 4 , These expansions then allow us to prove bounds on the low-energy portion of the evolution in Section 5. We prove bounds on the high-energy portion of the evolution in Section 6. Finally, we classify the threshold resonances and eigenfunctions in Section 7.

\section{Free RESOlVEnt EXPANSIONS AROUnd The THRESHOLd ENERGY $m$}

In this section we study the behavior of the free Dirac resolvent by using the properties of free Schrödinger resolvent operator $R_{0}(z)=(-\Delta-z)^{-1}$. Formally, the free resolvent

$$
R_{0}^{ \pm}\left(z^{2}\right)=\lim _{\epsilon \rightarrow 0^{+}}\left(-\Delta-\left(z^{2} \pm i \epsilon\right)\right)^{-1}
$$

and the perturbed resolvent operators

$$
R_{V}^{ \pm}\left(z^{2}\right)=\lim _{\epsilon \rightarrow 0^{+}}\left(-\Delta+V-\left(z^{2} \pm i \epsilon\right)\right)^{-1}
$$

are well-defined as an operator between weighted $L^{2}\left(\mathbb{R}^{2}\right)$ spaces, see [2].

Here we review some estimates (see e.g. [41, 23, 24]) for $R_{0}^{ \pm}\left(z^{2}\right)$ needed to study the Dirac evolution. To best utilize these expansions, we employ the notation

$$
f(z)=\widetilde{O}(g(z))
$$

to denote

$$
\frac{d^{j}}{d z^{j}} f=O\left(\frac{d^{j}}{d z^{j}} g\right), \quad j=0,1,2,3, \ldots
$$

The notation refers to derivatives with respect to the spectral variable $z$, or $|x-y|$ in the expansions for the integral kernel of the free resolvent operator, which is a function of the variable $\rho=z|x-y|$. If the derivative bounds hold only for the first $k$ derivatives we write $f=\widetilde{O}_{k}(g)$. In addition, if we write $f=\widetilde{O}_{k}(1)$, we mean that differentiation up to order $k$ is comparable to division by $z$ and/or $|x-y|$. This notation applies to operators as well as scalar functions; the meaning should be clear from the context.

Lemma 2.1. Let $0<z \ll 1$. For $z|x-y|<1$, we have the expansions

$$
\begin{aligned}
R_{0}^{ \pm}\left(z^{2}\right)=g^{ \pm}(z)+G_{0}+ & \widetilde{O}_{2}\left(z^{2}|x-y|^{2} \log (z|x-y|)\right) \\
& =g^{ \pm}(z)+G_{0}+g_{1}^{ \pm}(z) G_{1}+z^{2} G_{2}+\widetilde{O}_{2}\left(z^{4}|x-y|^{4} \log (z|x-y|)\right)
\end{aligned}
$$


where

$$
\begin{gathered}
g^{ \pm}(z)=-\frac{1}{2 \pi}(\log (z / 2)+\gamma) \pm \frac{i}{4} \\
g_{1}^{ \pm}(z)=-\frac{z^{2}}{4} g^{ \pm}(z)-\frac{z^{2}}{8 \pi} \\
G_{0} f(x)=-\frac{1}{2 \pi} \int_{\mathbb{R}^{2}} \log |x-y| f(y) d y \\
G_{1} f(x)=\int_{\mathbb{R}^{2}}|x-y|^{2} f(y) d y \\
G_{2} f(x)=\frac{1}{8 \pi} \int_{\mathbb{R}^{2}}|x-y|^{2} \log |x-y| f(x) d y
\end{gathered}
$$

For $z|x-y|>1$, we have

$$
R_{0}^{ \pm}\left(z^{2}\right)(x, y)=e^{ \pm i z|x-y|} \omega_{ \pm}(z|x-y|), \quad\left|\omega_{ \pm}^{(j)}(y)\right| \lesssim(1+|y|)^{-\frac{1}{2}-j}, \quad j=0,1,2, \ldots .
$$

We develop expansions on the positive portion of the spectrum, $[m, \infty)$. The negative branch, $(-\infty,-m]$, can be studied analogously with only minor changes, see Remark 7.4 below. We write $\lambda=\sqrt{m^{2}+z^{2}}$ with $0<z \ll 1$. Using (6) we have

$$
\begin{aligned}
\mathcal{R}_{0}^{ \pm}(\lambda)=\left[-i \alpha \cdot \nabla+m \beta+\sqrt{m^{2}+z^{2}} I\right] & R_{0}^{ \pm}\left(z^{2}\right)= \\
& {\left[-i \alpha \cdot \nabla+m(\beta+I)+\frac{z^{2}}{2 m} I+\widetilde{O}\left(z^{4}\right) I\right] R_{0}^{ \pm}\left(z^{2}\right) . }
\end{aligned}
$$

We now employ the following notational conventions. The operators $M_{11}$ and $M_{22}$ are defined to be matrix-valued operators with kernels

$$
M_{11}(x, y)=\left(\begin{array}{ll}
1 & 0 \\
0 & 0
\end{array}\right), \quad M_{22}(x, y)=\left(\begin{array}{ll}
0 & 0 \\
0 & 1
\end{array}\right) .
$$

We also define the projection operators $I_{1}, I_{2}$ by

$$
I_{1}\left(\begin{array}{l}
a \\
b
\end{array}\right)=\left(\begin{array}{l}
a \\
0
\end{array}\right), \quad I_{2}\left(\begin{array}{l}
a \\
b
\end{array}\right)=\left(\begin{array}{l}
0 \\
b
\end{array}\right) .
$$

Using (10) and (17), we have (for $z|x-y|<1,0<z \ll 1, \lambda=\sqrt{z^{2}+m^{2}}$ )

$$
\begin{aligned}
\mathcal{R}_{0}^{ \pm}(\lambda)=\left[-i \alpha \cdot \nabla+2 m I_{1}+\frac{z^{2}}{2 m} I+\widetilde{O}\left(z^{4}\right) I\right] \\
{\left[g^{ \pm}(z)+G_{0}+g_{1}^{ \pm}(z) G_{1}+z^{2} G_{2}+\widetilde{O}_{2}\left(z^{4}|x-y|^{4} \log (z|x-y|)\right)\right] . }
\end{aligned}
$$

We define

$$
\begin{aligned}
\mathcal{G}_{0} & =-i \alpha \cdot \nabla G_{0}+2 m G_{0} I_{1} \\
\mathcal{G}_{1} & =-i \alpha \cdot \nabla G_{1}+2 m G_{1} I_{1}-\frac{2}{m} M_{11}-\frac{2}{m} M_{22}
\end{aligned}
$$




$$
\mathcal{G}_{2}=-i \alpha \cdot \nabla G_{2}+2 m G_{2} I_{1}+\frac{1}{2 m} G_{0}-\frac{1}{4 \pi m} M_{11}-\frac{1}{4 \pi m} M_{22} .
$$

We further define the function $\log ^{-}(y):=-\log (y) \chi_{\{0<y<1\}}$. Using this notation, the expansion (18) can be written as

$$
\mathcal{R}_{0}^{ \pm}(\lambda)=2 m g^{ \pm}(z) M_{11}+\mathcal{G}_{0}+\widetilde{O}_{2}\left(z^{k}\left(|x-y|^{k}+\log ^{-}|x-y|\right)\right), \quad 0<k<2,
$$

or as

$$
\begin{aligned}
\mathcal{R}_{0}^{ \pm}(\lambda)=2 m g^{ \pm}(z) M_{11}+\mathcal{G}_{0}+g_{1}^{ \pm}(z) \mathcal{G}_{1}+ & z^{2} \mathcal{G}_{2} \\
& +\widetilde{O}_{2}\left(z^{\ell}\left(|x-y|^{\ell}+\log ^{-}|x-y|\right)\right), \quad 2<\ell<4 .
\end{aligned}
$$

To obtain these formulas, we write using (12) that

$$
\frac{z^{2}}{2 m} g^{ \pm}(z)=\frac{z^{2}}{2 m} g^{ \pm}(z)\left[M_{11}+M_{22}\right]=-\frac{z^{2}}{4 \pi m}\left[M_{11}+M_{22}\right]-\frac{2}{m} g_{1}^{ \pm}(z)\left[M_{11}+M_{22}\right]
$$

In this expansion we chose to group terms by their size with respect to the spectral variable $z$ rather than by operator.

Combining the expansions (22) and (23) with the high energy expansion (16), we obtain

Lemma 2.2. We have the following expansion for the kernel of the free resolvent, $\lambda=\sqrt{m^{2}+z^{2}}$, $0<z \ll 1$

$$
\mathcal{R}_{0}^{ \pm}(\lambda)(x, y)=2 m g^{ \pm}(z) M_{11}+\mathcal{G}_{0}(x, y)+E_{0}^{ \pm}(z)(x, y)
$$

where $E_{0}^{ \pm}$satisfies the bounds

$$
\left|E_{0}^{ \pm}\right| \lesssim z^{k}\left(|x-y|^{k}+\log ^{-}|x-y|\right),\left|\partial_{z} E_{0}^{ \pm}\right| \lesssim z^{k-1}\left(|x-y|^{k}+\log ^{-}|x-y|\right),
$$

for any $\frac{1}{2} \leq k<2$. Furthermore, we have

$$
E_{0}^{ \pm}(z)(x, y)=g_{1}^{ \pm}(z) \mathcal{G}_{1}+z^{2} \mathcal{G}_{2}+E_{1}^{ \pm}(z)(x, y),
$$

where

$$
\left|E_{1}^{ \pm}\right| \lesssim z^{\ell}\left(|x-y|^{\ell}+\log ^{-}|x-y|\right),\left|\partial_{z} E_{1}^{ \pm}\right| \lesssim z^{\ell-1}\left(|x-y|^{\ell}+\log ^{-}|x-y|\right),
$$

for any $2<\ell<4$.

Proof. For $z|x-y|<1$, we already obtained the required bound in (22).

For $z|x-y| \gtrsim 1$, using (16), we have (with $\lambda=\sqrt{m^{2}+z^{2}}$ )

$$
\begin{aligned}
\mathcal{R}_{0}^{ \pm}(\lambda)=\left[-i \alpha \cdot \nabla+m \beta+\sqrt{m^{2}+z^{2}} I\right] R_{0}^{ \pm}\left(z^{2}\right) & \\
= & {\left[-i \alpha \cdot \nabla+m \beta+\sqrt{m^{2}+z^{2}} I\right]\left[e^{ \pm i z|x-y|} \omega_{ \pm}(z|x-y|)\right] . }
\end{aligned}
$$

Therefore, for $z|x-y| \gtrsim 1$ and $0<z \ll 1$ (in this case $|x-y| \gtrsim 1$ ), we have

$$
\left|\partial_{z}^{j} \mathcal{R}_{0}^{ \pm}(\lambda)(x, y)\right| \lesssim z^{-1 / 2}|x-y|^{j-1 / 2}
$$


Also using

$$
E_{0}^{ \pm}(z)=\mathcal{R}_{0}^{ \pm}(\lambda)-2 m g^{ \pm}(z) M_{11}-\mathcal{G}_{0}
$$

we have for $j=0,1$,

$$
\left|\partial_{z}^{j} E_{0}^{ \pm}(z)(x, y) \chi_{\{z|x-y|>1\}}\right| \lesssim z^{-1 / 2}|x-y|^{j-1 / 2}+z^{-j}(z|x-y|)^{0+} .
$$

Now, note that for any $\frac{1}{2} \leq k<2$, and for $j=0,1$, we have

$$
\begin{aligned}
\left|\partial_{z}^{j} E_{0}^{ \pm}(z)(x, y)\right| \lesssim\left[z^{k-j}\left(|x-y|^{k}+\log ^{-}|x-y|\right) \chi_{\{z|x-y|<1\}}\right. & \\
\left.+\left(z^{-1 / 2}|x-y|^{j-1 / 2}+z^{-j}(z|x-y|)^{0+}\right) \chi_{\{z|x-y|>1\}}\right] & \\
& \lesssim z^{k-j}\left(|x-y|^{k}+\log ^{-}|x-y|\right) .
\end{aligned}
$$

The proof for $E_{1}^{ \pm}$is similar. We already obtained the required bound in the case $z|x-y|<1$ in (23). For $z|x-y|>1$, using the high energy estimate above, and

$$
E_{1}^{ \pm}=\mathcal{R}_{0}^{ \pm}(\lambda)-2 m g^{ \pm}(z) M_{11}-\mathcal{G}_{0}-g_{1}^{ \pm}(z) \mathcal{G}_{1}-z^{2} \mathcal{G}_{2}
$$

we obtain (note that $|x-y| \gtrsim 1$ )

$$
\left|\partial_{z}^{j} E_{1}^{ \pm}(z)(x, y) \chi_{\{z|x-y|>1\}}\right| \lesssim z^{-1 / 2}|x-y|^{j-1 / 2}+z^{-j}(z|x-y|)^{2+} \lesssim z^{-j}(z|x-y|)^{2+} .
$$

Hence, for any $2<\ell<4$ and $j=0,1$, we have

$$
\begin{array}{r}
\left|\partial_{z}^{j} E_{1}^{ \pm}(z)(x, y)\right| \lesssim\left[z^{\ell-j}\left(|x-y|^{\ell}+\log ^{-}|x-y|\right) \chi_{\{z|x-y|<1\}}+z^{-j}(z|x-y|)^{2+} \chi_{\{z|x-y|>1\}}\right] \\
\lesssim z^{\ell-j}\left(|x-y|^{\ell}+\log ^{-}|x-y|\right)
\end{array}
$$

\section{Free Dirac Dispersive estimates}

Due to the relationship between the free Dirac evolution and the free Klein-Gordon equation, $D_{m}^{2} f=\left(-\Delta+m^{2}\right) f$, we can expect a natural time decay rate of size $|t|^{-\frac{1}{2}}$ as one has in the wave equation (when $m=0$ ) or Klein-Gordon equation (when $m>0$ ), provided the initial data has more than $\frac{3}{2}$ weak derivatives in $L^{1}\left(\mathbb{R}^{2}\right)$. In the case of Dirac equation, as in Klein-Gordon, the time decay can be improved to $\langle t\rangle^{-1}$ for smoother initial data. In particular, we have the following theorem bounding the evolution between classical Besov spaces.

Theorem 3.1. Fix $j \in \mathbb{N}$. Let $\chi_{j}$ be a smooth cut-off for the set $\lambda \approx 2^{j}$. Then, the free Dirac equation satisfies:

$$
\left\|e^{-i t D_{m}} \chi_{j}\left(D_{m}\right) f\right\|_{L^{\infty}} \lesssim \min \left(2^{2 j}, 2^{\frac{3 j}{2}}|t|^{-1 / 2}, 2^{2 j}|t|^{-1}\right)\|f\|_{L^{1}} .
$$

Let $\chi_{0}(\lambda)$ be a smooth cut-off for a small neighborhood of $m$. Then, we have

$$
\left\|e^{-i t D_{m}} \chi_{0}\left(D_{m}\right) f\right\|_{L^{\infty}} \lesssim \frac{1}{\langle t\rangle}\|f\|_{L^{1}}
$$


This estimate can best be viewed as a mapping on Besov spaces 4

Corollary 3.2. Under the assumptions of Theorem 3.1, for any $\theta \in\left[0, \frac{1}{2}\right]$ and $s-s^{\prime} \geq \frac{3}{2}+\theta$, we have the following bounds

$$
\left\|e^{-i t D_{m}}\right\|_{B_{1,1}^{s} \rightarrow B_{\infty, 1}^{s^{\prime}}} \leq C\left\{\begin{array}{cc}
|t|^{-\frac{1}{2}+\theta} & 0<|t|<1 \\
|t|^{-\frac{1}{2}-\theta} & |t| \geq 1
\end{array}\right.
$$

First note that (with $\lambda=\sqrt{z^{2}+m^{2}}$ )

$$
\int_{m}^{\infty} e^{-i t \lambda} \chi_{j}(\lambda)\left[\mathcal{R}_{0}^{+}(\lambda)-\mathcal{R}_{0}^{-}(\lambda)\right] d \lambda=\int_{0}^{\infty} e^{-i t \sqrt{z^{2}+m^{2}}} \frac{z \chi_{j}(z)\left[\mathcal{R}_{0}^{+}(\lambda)-\mathcal{R}_{0}^{-}(\lambda)\right]}{\sqrt{z^{2}+m^{2}}} d z .
$$

Using (17), the formula $\left[R_{0}^{+}-R_{0}^{-}\right]\left(z^{2}\right)(x, y)=\frac{i}{2} J_{0}(z|x-y|)$, and the asymptotics for the Bessel function, see [1], we can write

$$
\begin{aligned}
& \frac{\mathcal{R}_{0}^{+}(\lambda)-\mathcal{R}_{0}^{-}(\lambda)}{\sqrt{z^{2}+m^{2}}}=\frac{\left(-i \alpha \cdot \nabla+m \beta+\sqrt{z^{2}+m^{2}} I\right)}{\sqrt{z^{2}+m^{2}}}\left[R_{0}^{+}-R_{0}^{-}\right]\left(z^{2}\right)(x, y) \\
& = \begin{cases}\widetilde{O}_{1}\left(z / \sqrt{z^{2}+m^{2}}\right) & z|x-y| \ll 1 \\
e^{i z|x-y| \widetilde{\omega}_{+}(z|x-y|)+e^{-i z|x-y|} \widetilde{\omega}_{-}(z|x-y|)} & z|x-y| \gtrsim 1\end{cases}
\end{aligned}
$$

where $\widetilde{\omega}_{ \pm}(z|x-y|)$ satisfies the same properties as $\omega_{ \pm}(z|x-y|)$ in (16). Therefore, it suffices to consider the integrals

$$
\int_{0}^{\infty} e^{-i t \sqrt{z^{2}+m^{2}}} z \chi_{j}(z) \widetilde{O}_{1}\left(z / \sqrt{z^{2}+m^{2}}\right) d z
$$

and

$$
\int_{0}^{\infty} e^{-i t \sqrt{z^{2}+m^{2}} \pm i z|x-y|} z \chi_{j}(z) \widetilde{\omega}_{ \pm}(z|x-y|) \widetilde{\chi}(z|x-y|) d z
$$

The integral (26) is $O\left(\min \left(2^{2 j}, 2^{j} /|t|\right)\right)$. The first bound follows since the integrand is bounded by $z \chi_{j}(z)$, and the second bound follows from an integration by parts. To estimate the integral in (27), we apply stationary phase method using the following (slightly modified) lemma from [41,

Lemma 3.3. Let $\phi^{\prime}\left(z_{0}\right)=0$ and $1 \leq \phi^{\prime \prime} \leq C$. Then,

$$
\left|\int_{-\infty}^{\infty} e^{-i t \phi(z)} a(z) d z\right| \lesssim \int_{\left|z-z_{0}\right|<|t|^{-\frac{1}{2}}}|a(z)| d z+|t|^{-1} \int_{\left|z-z_{0}\right|>|t|^{-\frac{1}{2}}}\left(\frac{|a(z)|}{\left|z-z_{0}\right|^{2}}+\frac{\left|a^{\prime}(z)\right|}{\left|z-z_{0}\right|}\right) d z .
$$

\footnotetext{
${ }^{4}$ For $s \in \mathbb{R}$ and $1 \leq p, q \leq \infty$, we define the Besov space $B_{p, q}^{s}\left(\mathbb{R}^{2}, \mathbb{C}^{2}\right)$ to be the space of all tempered distributions $f$ such that

$$
\|f\|_{B_{p, q}^{s}}=\left(\left\|P_{<1} f\right\|_{p}^{q}+\sum_{j=1}^{\infty} 2^{j s q}\left\|P_{j} f\right\|_{p}^{q}\right)^{\frac{1}{q}}<\infty,
$$
}

where $P_{<1}$ and $P_{j}$ are the usual Littlewood-Paley projections. 
Using Lemma 3.3 with $\phi_{ \pm}(z)=\sqrt{z^{2}+m^{2}} \mp \frac{z r}{t}, r:=|x-y|$, we will prove the following lemma which yields the desired bound for the integral in (27) for $j=0$. Let $\tilde{\chi}$ be a smooth cut-off for $[1, \infty)$ supported in $(1 / 2, \infty)$.

Lemma 3.4. If

$$
|a(z)| \lesssim \frac{z \chi(z) \widetilde{\chi}(z r)}{(1+z r)^{\frac{1}{2}}}, \quad\left|\partial_{z} a(z)\right| \lesssim \frac{\chi(z) \widetilde{\chi}(z r)}{(1+z r)^{\frac{1}{2}}},
$$

then we have the bound

$$
\left|\int_{0}^{\infty} e^{-i t \phi_{ \pm}(z)} a(z) d z\right| \lesssim\langle t\rangle^{-1}
$$

where $\phi_{ \pm}(z)=\sqrt{z^{2}+m^{2}} \mp \frac{z r}{t}$.

Proof. The integral in question is clearly bounded. We need only show that for large $t$, the integral can be bounded by $|t|^{-1}$. We assume $t>0$ and treat only the case of $\phi_{+}$, in which case the critical point occurs in $[0, \infty)$. The case of $t<0$ can be treated with the argument below by interchanging the phases $\phi_{ \pm}$. Note that the critical point of $\phi_{+}$occurs at $z_{0}=m r / \sqrt{t^{2}-r^{2}}$. From this, we can assume that $t>2 r$, say, for $z_{0}$ to be in a small neighborhood of the support of $\chi(z)$. Thus, we have $z_{0} \approx r / t$.

We employ Lemma 3.3. First, consider the integral

$$
\int_{\left|z-z_{0}\right|<t^{-\frac{1}{2}}}|a(z)| d z \lesssim \int_{\left|z-z_{0}\right|<t^{-\frac{1}{2}}} \frac{z^{\frac{1}{2}} \widetilde{\chi}(z r)}{r^{\frac{1}{2}}} d z .
$$

We consider cases based on the size of $z_{0}$ compared to $t^{-\frac{1}{2}}$. First, consider the case when $z_{0} \gtrsim t^{-\frac{1}{2}}$. Then, we have $z \lesssim z_{0} \approx r / t$, and hence

$$
(28) \lesssim t^{-\frac{1}{2}} \frac{z_{0}^{\frac{1}{2}}}{r^{\frac{1}{2}}} \lesssim t^{-1}
$$

In the second case, we have $z_{0} \lesssim t^{-\frac{1}{2}}$, which yields $z \lesssim t^{-\frac{1}{2}}$. In this case, we see

$$
(28) \lesssim \int_{0}^{t^{-\frac{1}{2}}} \frac{z^{\frac{1}{2}} \tilde{\chi}(z r)}{r^{\frac{1}{2}}} d z \lesssim \frac{t^{-\frac{3}{4}}}{r^{\frac{1}{2}}} .
$$

We also note that the integral is zero unless $r \gtrsim t^{1 / 2}$, which provides the desired bound of $t^{-1}$.

We now proceed to bound the contribution of

$$
\int_{\left|z-z_{0}\right|>t^{-\frac{1}{2}}} \frac{|a(z)|}{\left|z-z_{0}\right|^{2}} d z \lesssim \int_{\left|z-z_{0}\right|>t^{-\frac{1}{2}}} \frac{z^{\frac{1}{2}} \widetilde{\chi}(z r)}{r^{\frac{1}{2}}\left|z-z_{0}\right|^{2}} d z .
$$

We only need show that this integral is bounded.

We first consider the case when $z_{0} \ll t^{-\frac{1}{2}}$, in which case we have $\left|z-z_{0}\right| \approx z$, and hence

$$
(29) \lesssim \int_{\mathbb{R}} \frac{\tilde{\chi}(z r)}{z^{\frac{3}{2}} r^{\frac{1}{2}}} d z \lesssim 1
$$


In the second case, when $t^{-\frac{1}{2}} \lesssim z_{0}$, since $z_{0} \approx r / t$, we have $r \gtrsim t^{\frac{1}{2}}$. With the change of variable $s=z+z_{0}$, we have

$$
(29) \lesssim \frac{1}{r^{\frac{1}{2}}} \int_{|s|>t^{-\frac{1}{2}}} \frac{s^{\frac{1}{2}}+z_{0}^{\frac{1}{2}}}{s^{2}} d s \lesssim r^{-\frac{1}{2}} t^{\frac{1}{4}}+r^{-\frac{1}{2}} t^{\frac{1}{2}} z_{0}^{\frac{1}{2}} \lesssim 1 .
$$

Finally, we turn to the contribution of

$$
\int_{\left|z-z_{0}\right|>t^{-\frac{1}{2}}} \frac{\left|a^{\prime}(z)\right|}{\left|z-z_{0}\right|} d z \lesssim \int_{\left|z-z_{0}\right|>t^{-\frac{1}{2}}} \frac{\tilde{\chi}(z r)}{z^{\frac{1}{2}} r^{\frac{1}{2}}\left|z-z_{0}\right|} d z
$$

If $z_{0} \ll t^{-\frac{1}{2}}$, we see that $\left|z-z_{0}\right| \approx z$ and similar to the treatment for (29) in this case, we have

$$
(30) \lesssim \int_{\mathbb{R}} \frac{\tilde{\chi}(z r)}{z^{\frac{3}{2}} r^{\frac{1}{2}}} d z \lesssim 1
$$

If $z_{0} \gtrsim t^{-\frac{1}{2}}$, we have $r \gtrsim t^{1 / 2}$ as above. We calculate

$$
(30) \lesssim \frac{1}{r^{\frac{1}{2}}}\left[\int_{\left|z-z_{0}\right|>t^{-\frac{1}{2}}} \frac{d z}{\left|z-z_{0}\right|^{\frac{3}{2}}}+\int_{\mathbb{R}} \frac{\tilde{\chi}(z r)}{z^{\frac{3}{2}}} d z\right] \lesssim r^{-\frac{1}{2}} t^{\frac{1}{4}}+1 \lesssim 1 .
$$

For completeness, we note that in the case of the phase $\phi_{-}$, the critical point occurs outside of $[0, \infty)$, and we have $\phi_{-}^{\prime}(z)=\frac{z}{\sqrt{z^{2}+m^{2}}}+\frac{r}{t} \gtrsim z$, we have

$$
\left|\int_{0}^{\infty} e^{-i t \phi_{-}(z)} a(z) d z\right| \lesssim t^{-1}\left(\int_{0}^{\infty} \frac{|a(z)|}{\left|\phi_{-}^{\prime}(z)\right|^{2}}+\frac{\left|a^{\prime}(z)\right|}{\left|\phi_{-}^{\prime}(z)\right|} d z\right) .
$$

Using the bounds for $a(z)$ and $a^{\prime}(z)$, we may bound this by

$$
t^{-1} \int_{0}^{\infty} \frac{\widetilde{\chi}(z r)}{z^{\frac{3}{2}} r^{\frac{1}{2}}} d z \lesssim t^{-1}
$$

as desired. This also takes care of the case when the critical point occurs outside a neighborhood of the support of $\chi$.

The following lemma finishes the proof of Theorem 3.1 by establishing the required bound for the integral in (27) when $j \geq 1$.

Lemma 3.5. Fix $j \in \mathbb{N}$, and let $\chi_{j}(z)$ be a cut-off to $z \approx 2^{j}$. If

$$
|a(z)| \lesssim \frac{z \chi_{j}(z) \widetilde{\chi}(z r)}{(1+z r)^{\frac{1}{2}}}, \quad\left|\partial_{z} a(z)\right| \lesssim \frac{\chi_{j}(z) \widetilde{\chi}(z r)}{(1+z r)^{\frac{1}{2}}},
$$

then we have the bound

$$
\left|\int_{0}^{\infty} e^{-i t \phi_{ \pm}(z)} a(z) d z\right| \lesssim \min \left(2^{2 j}, 2^{\frac{3 j}{2}}|t|^{-1 / 2}, 2^{2 j}|t|^{-1}\right),
$$

where $\phi_{ \pm}(z)=\sqrt{z^{2}+m^{2}} \mp \frac{z r}{t}$. 
Proof. First note that the integral is bounded by $2^{2 j}$ since the integrand is bounded by $z \chi_{j}(z)$. We now restrict ourselves to the case $t>0$ and only consider $\phi_{+}$. We also take $m=1$ without loss of generality. Let $\rho=2^{-j} z, q=2^{j} r$. We rewrite the integral as

$$
2^{2 j} \int_{0}^{\infty} e^{-i t 2^{-j} \widetilde{\phi}(\rho)} \widetilde{a}(\rho) d \rho
$$

where $\widetilde{\phi}(\rho)=2^{j}\left(\sqrt{2^{2 j} \rho^{2}+1}-\frac{\rho q}{t}\right)$. Moreover, $\widetilde{a}$ satisfies the bounds that $a$ satisfies with $j=1$ and $r=q$. Also note that $\frac{d^{2}}{d \rho^{2}} \widetilde{\phi} \approx 1$, since $\chi_{1}$ is supported away from 0 .

The critical point of $\widetilde{\phi}$ is $\rho_{0}=\frac{2^{-j} q}{\sqrt{t^{2} 2^{2 j}-q^{2}}}$. Therefore, $\rho_{0}$ is in the support of $\chi_{1}(\rho)$ provided that

$$
\left|t-q 2^{-j}\right| \approx q 2^{-3 j}
$$

which implies that $q \approx 2^{j} t$. This implies that

$$
|\widetilde{a}(\rho)| \lesssim \frac{\chi_{1}(\rho) \widetilde{\chi}(\rho q)}{\sqrt{2^{j} t}}, \quad\left|\partial_{\rho} \widetilde{a}(\rho)\right| \lesssim \frac{\chi_{1}(\rho) \widetilde{\chi}(\rho q)}{\sqrt{2^{j t}}}
$$

Using the first inequality above in (31) directly, we can bound (31) by $2^{3 j / 2} t^{-1 / 2}$. On the other hand, using these bounds in Lemma 3.3 with $t 2^{-j}$ instead of $t$. We bound (31) by

$$
2^{2 j} \int_{\left|\rho-\rho_{0}\right|<\sqrt{2^{j} / t}} \frac{1}{\sqrt{2^{j} t}} d \rho+\frac{2^{3 j}}{t} \int_{\left|\rho-\rho_{0}\right|>\sqrt{2^{j} / t}} \frac{1}{\sqrt{2^{j} t}}\left(\frac{1}{\left|\rho-\rho_{0}\right|^{2}}+\frac{\chi_{1}(\rho)}{\left|\rho-\rho_{0}\right|}\right) d \rho \lesssim \frac{2^{2 j}}{t} .
$$

In the cases $q \ll 2^{j} t$ or $q \gg 2^{j} t$, we have $\left|\frac{d}{d \rho} \widetilde{\phi}\right| \gtrsim 2^{2 j}$. Therefore, an integration by parts implies that the integral is bounded by $2^{j} / t$.

\section{Perturbed Resolvent expansions around the threshold energy $m$}

In this section, using Lemma 2.2, we develop expansions for the perturbed resolvents $\mathcal{R}_{V}^{ \pm}(\lambda)$ near the threshold $\lambda=m$ in the case when the threshold is regular, and when there is an s-wave resonance at the threshold, see Definition 4.3,

Since the matrix $V: \mathbb{R}^{2} \rightarrow \mathbb{C}^{2}$ is self-adjoint, the spectral theorem allows us to write

$$
V=B^{*}\left(\begin{array}{cc}
\lambda_{1} & 0 \\
0 & \lambda_{2}
\end{array}\right) B
$$

with $\lambda_{j} \in \mathbb{R}$. We further write $\eta_{j}=\left|\lambda_{j}\right|^{\frac{1}{2}}$,

$$
V=B^{*}\left(\begin{array}{cc}
\eta_{1} & 0 \\
0 & \eta_{2}
\end{array}\right) U\left(\begin{array}{cc}
\eta_{1} & 0 \\
0 & \eta_{2}
\end{array}\right) B=v^{*} U v
$$

where

$$
U=\left(\begin{array}{cc}
\operatorname{sign}\left(\lambda_{1}\right) & 0 \\
0 & \operatorname{sign}\left(\lambda_{2}\right)
\end{array}\right), \quad \text { and } v=\left(\begin{array}{cc}
a & b \\
c & d
\end{array}\right):=\left(\begin{array}{cc}
\eta_{1} & 0 \\
0 & \eta_{2}
\end{array}\right) B
$$


Note that the entries of $v$ are $\lesssim\langle x\rangle^{-\beta / 2}$, provided that the entries of $V$ are $\lesssim\langle x\rangle^{-\beta}$. This representation of $V$ allows us to employ the symmetric resolvent identity to write the perturbed resolvent $\mathcal{R}_{V}(\lambda)=\left(D_{m}+V-\lambda\right)^{-1}$ as (with $\left.\lambda=\sqrt{m^{2}+z^{2}}, 0<z \ll 1\right)$

$$
\mathcal{R}_{V}(\lambda)=\mathcal{R}_{0}(\lambda)-\mathcal{R}_{0}(\lambda) v^{*}\left(U+v \mathcal{R}_{0}(\lambda) v^{*}\right)^{-1} v \mathcal{R}_{0}(\lambda) .
$$

Our goal is to invert the operator

$$
M^{ \pm}(z)=U+v \mathcal{R}_{0}^{ \pm}\left(\sqrt{m^{2}+z^{2}}\right) v^{*}, \quad 0<z \ll 1 .
$$

Recall that $\mathcal{R}_{0}(\lambda)(x, y)=2 m g^{ \pm}(z) M_{11}+\mathcal{G}_{0}(x, y)+E_{0}^{ \pm}(z)(x, y)$. Therefore,

$$
M^{ \pm}(z)=U+v \mathcal{G}_{0} v^{*}+2 m g^{ \pm}(z) v M_{11} v^{*}+v E_{0}^{ \pm}(z) v^{*} .
$$

Recalling (32), for $f=\left(f_{1}, f_{2}\right)^{T} \in L^{2} \times L^{2}$, we have

$$
v M_{11} v^{*} f(x)=\left(\begin{array}{c}
a(x) \\
c(x)
\end{array}\right) \int_{\mathbb{R}^{2}} \bar{a}(y) f_{1}(y)+\bar{c}(y) f_{2}(y) d y .
$$

Thus, we arrive at

$$
v M_{11} v^{*}=\|(a, c)\|_{2}^{2} P,
$$

where $P$ is the projection onto the vector $(a, c)^{T}$. We also define the operators $Q:=1-P$, $T:=U+v \mathcal{G}_{0} v^{*}$, and let

$$
\mathrm{g}^{ \pm}(z):=2 m\|(a, c)\|_{2}^{2} g^{ \pm}(z)
$$

We have

Lemma 4.1. For $0<z \ll 1$, we have

$$
M^{ \pm}(z)=\mathrm{g}^{ \pm}(z) P+T+M_{0}^{ \pm}(z)
$$

where 5 for any $\frac{1}{2} \leq k<2$,

$$
\left\|\sup _{0<z \ll 1} z^{j-k}\left|\partial_{z}^{j} M_{0}^{ \pm}(z)(x, y)\right|\right\|_{H S} \lesssim 1, \quad j=0,1,
$$

if $\left|v_{i j}(x)\right| \lesssim\langle x\rangle^{-\beta}$ for some $\beta>1+k$.

Moreover,

$$
M_{0}^{ \pm}(z)=g_{1}^{ \pm}(z) v \mathcal{G}_{1} v^{*}+z^{2} v \mathcal{G}_{2} v^{*}+M_{1}^{ \pm}(z),
$$

where, for any $2<\ell<4$,

$$
\left\|\sup _{0<z \ll 1} z^{j-\ell}\left|\partial_{z}^{j} M_{1}^{ \pm}(z)(x, y)\right|\right\|_{H S} \lesssim 1, \quad j=0,1,
$$

${ }^{5}$ The Hilbert-Schmidt norm of an integral operator $K$ with integral kernel $K(x, y)$ is defined by

$$
\|K\|_{H S}^{2}=\int_{\mathbb{R}^{4}}|K(x, y)|^{2} d x d y .
$$


if $\beta>1+\ell$.

Proof. Note that by (34), Lemma 2.2 , and the discussion above, we have

$$
M_{0}=v E_{0} v^{*}, M_{1}=v E_{1} v^{*} .
$$

Therefore the statement for $j=0,1$ follows from the error bounds in Lemma 2.2, and the fact that $\left(|x-y|^{\ell}+\log ^{-}|x-y|\right)\langle x\rangle^{-\beta}\langle y\rangle^{-\beta}$ is a Hilbert-Schmidt kernel for $\beta>1+\ell$ and $\ell>-1$.

We employ the following terminology, following [41, 23, 24]

Definition 4.2. We say an operator $T: L^{2} \times L^{2}\left(\mathbb{R}^{2}\right) \rightarrow L^{2} \times L^{2}\left(\mathbb{R}^{2}\right)$ with kernel $T(\cdot, \cdot)$ is absolutely bounded if the operator with kernel $|T(\cdot, \cdot)|$ is bounded from $L^{2} \times L^{2}\left(\mathbb{R}^{2}\right)$ to $L^{2} \times L^{2}\left(\mathbb{R}^{2}\right)$.

We note that Hilbert-Schmidt and finite-rank operators are absolutely bounded operators.

As in the case of the Schrödinger operator, the invertibility of the leading term of $M$ depends on the regularity of the threshold energy. Here we give the definition of threshold resonances. Later, in Section 7, we study the classification of these resonances in detail.

Definition 4.3. (1) Let $Q=1-P$. We say that $\lambda=m$ is a regular point of the spectrum of $H=D_{m}+V$ provided that $Q T Q=Q\left(U+v \mathcal{G}_{0} v^{*}\right) Q$ is invertible on $Q\left(L^{2} \times L^{2}\right)$. If $Q T Q$ is invertible, we denote $D_{0}:=(Q T Q)^{-1}$ as an operator on $Q\left(L^{2} \times L^{2}\right)$.

(2) Assume that $m$ is not a regular point of the spectrum. Let $S_{1}$ be the Riesz projection onto the kernel of $Q T Q$ as an operator on $Q\left(L^{2} \times L^{2}\right)$. Then $Q T Q+S_{1}$ is invertible on $Q\left(L^{2} \times L^{2}\right)$. Accordingly, with a slight abuse of notation we redefine $D_{0}=\left(Q T Q+S_{1}\right)^{-1}$ as an operator on $Q\left(L^{2} \times L^{2}\right)$. We say there is a resonance of the first kind at $m$ if the operator $T_{1}:=S_{1} T P T S_{1}$ is invertible on $S_{1}\left(L^{2} \times L^{2}\right)$.

(3) We say there is a resonance of the second kind at $m$ if $T_{1}$ is not invertible on $S_{1}\left(L^{2} \times L^{2}\right)$ but $T_{2}:=S_{2} v \mathcal{G}_{1} v^{*} S_{2}$ is invertible on $S_{2}\left(L^{2} \times L^{2}\right)$, where $S_{2}$ is the Riesz projection onto the kernel of $T_{1}$. Recall the definition of $\mathcal{G}_{1}$ and $\mathcal{G}_{2}$ in (14) and (15).

(4) Finally, if $T_{2}$ is not invertible on $S_{2}\left(L^{2} \times L^{2}\right)$, we say there is a resonance of the third kind at $m$. We note that in this case the operator $T_{3}:=S_{3} v \mathcal{G}_{2} v^{*} S_{3}$ is always invertible on $S_{3}\left(L^{2} \times L^{2}\right)$, where $S_{3}$ is the Riesz projection onto the kernel of $T_{2}$ (see Lemma 7.8 below).

Remark 4.4. i) Since $S_{1} \leq Q$, for any $\phi \in S_{1}, P \phi=0$, i.e.

$$
M_{11} v^{*} \phi=0 .
$$

ii) Note that $v \mathcal{G}_{0} v^{*}$ is compact and self-adjoint. Hence, QTT is a compact perturbation of QUQ and it is self-adjoint. Also, the spectrum of $Q U Q$ is in $\{-1,1\}$. Hence, zero is the isolated point of the spectrum of $Q T Q$ and $\operatorname{dim}\left(\operatorname{Ker}_{Q T Q}\right)$ is finite. Thus $S_{1}$ is a finite rank projection.

iii) As in the case of Schrödinger operator in $\mathbb{R}^{2}$ (see e.g. [35]), the projections $S_{1}-S_{2}, S_{2}-S_{3}$ 
and $S_{3}$ correspond to s-wave resonances, p-wave resonances, and eigenspace at $m$ respectively. In particular, resonance of the first kind means that there is only an s-wave resonance at $m$. Resonance of the second kind means that there is a p-wave resonance, and there may or may not be an s-wave resonance. Finally, resonance of the third kind means that $m$ is an eigenvalue, and there may or may not be s-wave and p-wave resonances. We characterize these projections in Section 17. We will also prove, see Remark 7.7, that the rank of $S_{1}-S_{2}$ is at most 1 and the rank of $S_{2}-S_{3}$ is at most 2 .

iv) Since $Q T Q$ is self-adjoint, $S_{1}$ is the orthogonal projection onto the kernel of $Q T Q$, and we have (with $\left.D_{0}=\left(Q T Q+S_{1}\right)^{-1}\right)$

$$
S_{1} D_{0}=D_{0} S_{1}=S_{1}
$$

This statement also valid for $S_{2}$ and $\left(T_{1}+S_{2}\right)^{-1}$, and for $S_{3}$ and $\left(T_{2}+S_{3}\right)^{-1}$.

v) The operator $Q D_{0} Q$ is absolutely bounded in $L^{2} \times L^{2}$, see Lemma 7.1 below.

vi) The operators with kernel $v \mathcal{G}_{k} v^{*}$ are Hilbert-Schmidt operators on $L^{2} \times L^{2}$ if $\left|v_{i j}(x)\right| \lesssim\langle x\rangle^{-\beta}$ for $\beta>\frac{3}{2}$ if $k=1$ and $\beta>3$ for $k=2,3$. However, $v \mathcal{G}_{0} v^{*}$ is not Hilbert-Schmidt because of the local singularity of size $|x-y|^{-1}$.

We can now use the expansions for $M^{-1}$ from the papers [41, 23, 24] since $M$ has the same form with the same error bounds, and with analogous definitions for $S_{j}$. We include these expansions without proof.

Lemma 4.5. Assume that $m$ is a regular point of the spectrum of $H$. Also assume that $\left|v_{i j}(x)\right| \lesssim$ $\langle x\rangle^{-\frac{3}{2}-}$. Then

$$
\left(M^{ \pm}(z)\right)^{-1}=h^{ \pm}(z)^{-1} S+Q D_{0} Q+E^{ \pm}(z)
$$

where

$$
S=\left[\begin{array}{cc}
P & -P T Q D_{0} Q \\
-Q D_{0} Q T P & Q D_{0} Q T P T Q D_{0} Q
\end{array}\right]
$$

$h^{ \pm}(z)=\mathrm{g}^{ \pm}(z)+$ trace $\left(P T P-P T Q D_{0} Q T P\right), S$ is a self-adjoint, finite rank operator, and

$$
\left\|\sup _{0<z \ll 1} z^{j-1 / 2}\left|\partial_{z}^{j} E^{ \pm}(z)(x, y)\right|\right\|_{H S} \lesssim 1, \quad j=0,1,
$$

Lemma 4.6. Assume that there is a resonance at $m$ of the first kind. Also assume that $\left|v_{i j}(x)\right| \lesssim$ $\langle x\rangle^{-\frac{3}{2}-}$. Then

$$
\begin{aligned}
M^{ \pm}(z)^{-1}=-h_{ \pm}(z) S_{1} D_{1} S_{1}-S S_{1} D_{1} & S_{1}-S_{1} D_{1} S_{1} S \\
& -h_{ \pm}(z)^{-1} S S_{1} D_{1} S_{1} S+h_{ \pm}(z)^{-1} S+Q D_{0} Q+E^{ \pm}(z),
\end{aligned}
$$

Here $E^{ \pm}(z), S$, and $h^{ \pm}(z)$ are as in the previous lemma with $D_{0}=Q\left(T+S_{1}\right)^{-1} Q$, and $D_{1}=$ $T_{1}^{-1}=\left(S_{1} T P T S_{1}\right)^{-1}$. 
Remark 4.7. One can also obtain analogous espansion 6 in the cases when there is a resonance of the second or third kind as in the Schrödinger equation, [23]. We chose not to state these expansions explicitly since we are not considering dispersive estimates in these cases. By substituting the expansions for $M^{ \pm}(z)^{-1}$ in Lemmas 4.5 and 4.6 into (33), we obtain expansions for the resolvent showing that $(\lambda-m) \mathcal{R}_{V}(\lambda)$ is uniformly bounded between weighted $L^{2}$ spaces in a neighborhood of $m$. This implies that there are no eigenvalues in a neighborhood of $m$. In particular, there are only finitely many eigenvalues in the spectral gap $(-m, m)$. It also implies a limiting absorption principle bound around the threshold.

\section{LOW ENERGY DISPERSIVE ESTIMATES}

In this section we study the low-energy part of the perturbed Dirac evolution. For technical reasons, which we detail below, we consider the evolution as an operator from $H^{1}$ to $B M O$.

Theorem 5.1. Under Assumption 1.1 part i), with $\chi$ a smooth cut-off to a sufficiently small neighborhood of the threshold energy $\lambda=m$. We have the dispersive bound

$$
\left\|e^{-i t H} P_{a c}(H) \chi(H) f\right\|_{B M O} \lesssim \frac{1}{\langle t\rangle}\|f\|_{H^{1}} .
$$

This bound holds if $\lambda=m$ is regular or if there is a resonance of the first kind.

As usual, we prove this bound by considering the Stone's formula, (9). In the case there is a resonance at $m$ of the first kind, using Lemma 4.6 in (33), we have

$$
\begin{aligned}
\mathcal{R}_{V}(\lambda)= & \mathcal{R}_{0}(\lambda) \\
& -\mathcal{R}_{0}(\lambda) v^{*}\left[-h_{ \pm}(z) S_{1} D_{1} S_{1}+A+h_{ \pm}(z)^{-1}\left(S-S S_{1} D_{1} S_{1} S\right)+E^{ \pm}(z)\right] v \mathcal{R}_{0}(\lambda)
\end{aligned}
$$

where $A:=Q D_{0} Q-S S_{1} D_{1} S_{1}-S_{1} D_{1} S_{1} S$. Since this expansion contains the terms arising in the regular case, it suffices to prove the dispersive estimate in the case of a resonance of the first kind. We bound the contribution of each operator in this expansion in a series of technical propositions. The first term containing only a single free resolvent $\mathcal{R}_{0}$ is controlled by the bound in Theorem 3.1, specifically (25).

To control the contributions to the Stone's formula, using (17), (10), and (16), we write the outermost resolvents when $0<z \ll 1$ as

$$
\begin{aligned}
\mathcal{R}_{0}^{ \pm}(\lambda)(x, y)= & {[-i \alpha \cdot \nabla+e(z)] R_{0}^{ \pm}\left(z^{2}\right)(x, y)+2 m I_{1} R_{0}^{ \pm}\left(z^{2}\right)(x, y) } \\
= & -\frac{1}{2 \pi} \chi(z|x-y|)[-i \alpha \cdot \nabla] \log (z|x-y|) \\
& +\chi(z|x-y|)[-i \alpha \cdot \nabla]\left(R_{0}^{ \pm}\left(z^{2}\right)(x, y)+\frac{1}{2 \pi} \log (z|x-y|)\right)
\end{aligned}
$$

\footnotetext{
${ }^{6}$ These expansions would require more decay from the potential then we have in Assumption 1.1
} 


$$
\begin{aligned}
& +\chi(z|x-y|) e(z) R_{0}^{ \pm}\left(z^{2}\right)(x, y) \\
& +\widetilde{\chi}(z|x-y|) e^{ \pm i z|x-y|} \omega_{1}^{ \pm}(z(x-y))+2 m I_{1} R_{0}^{ \pm}\left(z^{2}\right)(x, y) \\
= & : R_{1}+R_{2}^{ \pm}+R_{3}^{ \pm}+R_{4}^{ \pm}+R_{5}^{ \pm} .
\end{aligned}
$$

Here $e(z)=\widetilde{O}_{1}\left(z^{2}\right)$, and it does not have \pm dependence. Further, $\omega_{1}^{ \pm}(z(x-y))$ satisfies the same bounds as $z \omega^{ \pm}(z|x-y|)$. We note that these expansions differ slightly from those in Sections 2 and 4, as we tailor them to prove the dispersive bounds rather than to develop expansions for $M^{ \pm}(z)^{-1}$.

We note that the dispersive bounds for the term containing only $R_{5}^{ \pm}$is identical to the ones given for the Schrödinger operator in [41] and [23], since $R_{5}^{ \pm}$satisfies the same bounds and cancellation properties as the Schrödinger resolvent $R_{0}^{ \pm}$. Moreover, the corresponding orthogonality property

$$
Q v M_{11}=M_{11} v^{*} Q=0
$$

holds because of the projection $I_{1}$. The slight difference in the phase in Stone's formula can be taken care of using Lemma 3.4 in place of Lemma 2 in [41. The contribution of the terms containing $R_{2}^{ \pm}$and $R_{3}^{ \pm}$in addition to $R_{5}^{ \pm}$are easier since $R_{2}^{ \pm}$and $R_{3}^{ \pm}$satisfy the same bounds as $F$ or $G$ from Lemma 3.3 in [23] (also see [41]). Therefore one does not need the orthogonality property for these terms.

Thus, it suffices to consider the terms containing $R_{1}$ or $R_{4}^{ \pm}$on the left. We will write the operator $\mathcal{R}_{0}^{ \pm}$on the right as $\mathcal{R}_{L}^{ \pm}+\mathcal{R}_{H}^{ \pm}$, where

$$
\left\{\begin{array}{l}
\mathcal{R}_{L}^{ \pm}(\lambda)(x, y)=\chi(z|x-y|) \mathcal{R}_{0}^{ \pm}(\lambda)(x, y), \\
\mathcal{R}_{H}^{ \pm}(\lambda)(x, y)=\widetilde{\chi}(z|x-y|) \mathcal{R}_{0}^{ \pm}(\lambda)(x, y)=e^{ \pm i z|x-y|} \widetilde{\omega}_{ \pm}(z(x-y)) .
\end{array}\right.
$$

Before we bound the contribution of these terms to the Stone formula, (9), we note that the operator $R_{1}$ is not bounded as an operator from $L^{1} \rightarrow L^{2}$ or from $L^{2} \rightarrow L^{\infty}$. This is an important technical difference from the analysis of Schrödinger operators in [41, 23]. One can iterate the standard resolvent identity $\mathcal{R}_{V}=\mathcal{R}_{0}-\mathcal{R}_{0} V \mathcal{R}_{V}$ to smooth out the local singularity and obtain a bound from $L^{1} \rightarrow L^{\infty}$, though this would cause the time decay to be of the form $|t|^{-1}(\log t)^{k}$ for some $k>0$ for large $t$ due to the leading $\log \lambda$ behavior of the free resolvent, see Lemma 2.2, Instead, we consider the Dirac evolution as a mapping from the Hardy space $H^{1}$ to $B M O$. The following lemma is useful.

Lemma 5.2. For any $H^{1} \times H^{1}\left(\mathbb{R}^{2}\right)$ atom $g$, and for $0<z \lesssim 1$, we have

$$
v R_{1} g=g_{1}+g_{2} \widetilde{O}_{1}(z)
$$

where

$$
\left\|g_{j}\right\|_{L^{2}} \lesssim\|v\|_{L^{2}}+\|v\|_{L^{\infty}}, j=1,2
$$


Furthermore,

$$
\sup _{0<z \ll 1}\left(\left\|v \mathcal{R}_{L}^{ \pm} g\right\|_{L^{2}}+z\left\|v \partial_{z} \mathcal{R}_{L}^{ \pm} g\right\|_{L^{2}}\right) \lesssim\|v\|_{L^{2}}
$$

and

$$
\begin{gathered}
\left\|\chi(z)\left(\mathcal{R}_{L}^{+}-\mathcal{R}_{L}^{-}\right) g\right\|_{L_{x}^{\infty} L_{z}^{\infty}} \lesssim 1 \\
\left\|\chi(z) \partial_{z}\left(\mathcal{R}_{L}^{+}-\mathcal{R}_{L}^{-}\right) g\right\|_{L_{x}^{\infty} L_{z}^{1}} \lesssim 1
\end{gathered}
$$

Proof. We rewrite $R_{1}$ as

$$
\begin{aligned}
R_{1}=\frac{i}{2 \pi} \chi(z|x-y|) & \frac{\alpha \cdot(x-y)}{|x-y|^{2}} \\
& =\frac{i}{2 \pi} \frac{\alpha \cdot(x-y)}{|x-y|^{2}}-\frac{i}{2 \pi} \widetilde{\chi}(z|x-y|) \frac{\alpha \cdot(x-y)}{|x-y|^{2}}=\frac{i}{2 \pi} \frac{\alpha \cdot(x-y)}{|x-y|^{2}}+\widetilde{O}_{1}(z) .
\end{aligned}
$$

The contribution of the first summand gives $g_{1}$. By Theorem 1 in [20], the operator defined by the first term in $R_{1}$ is bounded from $H^{1}$ to $L^{2}$. Therefore,

$$
\left\|g_{1}\right\|_{L^{2}} \leq\|v\|_{L^{\infty}}\left\|\int_{\mathbb{R}^{2}} \frac{\alpha \cdot(x-y)}{|x-y|^{2}} g(y) d y\right\|_{L_{x}^{2}} \lesssim\|v\|_{L^{\infty}} .
$$

The bound for $g_{2}$ is immediate from the expansion above.

The second claim follows from the expansion

$$
\mathcal{R}_{L}(z)(x, y)=R_{1}-\frac{m I_{1}}{\pi} \log (z|x-y|) \chi(z|x-y|)+\widetilde{O}_{1}(1), \quad\left|\partial_{z} \mathcal{R}_{L}\right| \lesssim z^{-1},
$$

the fact that $\chi(z x) \log (z x) \in B M O$ with norm independent of $z$, and $H^{1}-B M O$ duality.

To obtain the last two claims, note that

$$
\begin{aligned}
{\left[\mathcal{R}_{L}^{+}-\mathcal{R}_{L}^{-}\right](\lambda)(x, y)=\chi(z|x-y|)\left[-i \alpha \cdot \nabla+m \beta+\sqrt{m^{2}+z^{2}} I\right] } & J_{0}(z|x-y|) \\
& =\chi(z|x-y|)\left(c I_{1}+\widetilde{O}_{1}(z)\right)
\end{aligned}
$$

which immediately implies (44). To obtain (45), note that

$$
\int\left(\int|x-y|\left|\chi^{\prime}(z|x-y|)\right| d z\right)\left|I_{1} g(y)\right| d y \lesssim\|g\|_{L^{1}}=1 .
$$

Recall that $\log ^{-}(y):=-\log (y) \chi_{\{0<y<1\}}$. In addition, we define $\log ^{+}(y)=\log (y) \chi_{\{y>1\}}$.

We start with the contribution of the terms $A:=Q D_{0} Q-S S_{1} D_{1} S_{1}-S_{1} D_{1} S_{1} S$ from (38), for which we rely only on the absolute boundedness of the operator, and do not use any orthogonality properties of the projection operators $Q$ or $S_{1} \leq Q$. By symmetry and the discussion above, it suffices to consider the terms

$$
\Gamma_{1}:=R_{1} v^{*} A v\left(\mathcal{R}_{L}^{+}-\mathcal{R}_{L}^{-}\right), \quad \Gamma_{2}:=R_{1} v^{*} A v \mathcal{R}_{H}^{+}, \quad \Gamma_{3}:=R_{4}^{+} v^{*} A v \mathcal{R}_{0}^{+},
$$


Proposition 5.3. Let $\Gamma_{j}$ be defined as in (48). Then, under the assumptions of Theorem 5.1, for any $H^{1} \times H^{1}\left(\mathbb{R}^{2}\right)$ atoms $f, g$, and for each $j=1,2,3$ we have

$$
\int_{0}^{\infty} e^{i t \sqrt{z^{2}+m^{2}}} \frac{z \chi(z)}{\sqrt{z^{2}+m^{2}}}\left\langle\Gamma_{j} f, g\right\rangle d z=O(1 /\langle t\rangle) .
$$

Proof. We start with $\Gamma_{1}$. By an integration by parts we rewrite the integral above as

$$
-\frac{i e^{i t m}}{t}\left\langle\left.\Gamma_{1}\right|_{z=0} f, g\right\rangle+\frac{i}{t} \int_{0}^{\infty} e^{i t \sqrt{z^{2}+m^{2}}} \partial_{z}\left[\chi(z)\left\langle\Gamma_{1} f, g\right\rangle\right] d z
$$

where $\left.\Gamma_{1}\right|_{z=0}$ means $\lim _{z \rightarrow 0+} \Gamma_{1}(z)$. Therefore, we need to prove that

$$
\left|\left\langle\left.\Gamma_{1}\right|_{z=0} f, g\right\rangle\right| \lesssim 1
$$

and

$$
\left\|\partial_{z}\left[\chi(z)\left\langle\Gamma_{1} f, g\right\rangle\right]\right\|_{L_{z}^{1}} \lesssim 1
$$

In fact, since $\chi(1)=0$, by the fundamental theorem of calculus, it suffices to prove (49). Using the bounds in Lemma 5.2, we have

$$
\left|\partial_{z}\left[\chi(z)\left\langle\Gamma_{1} f, g\right\rangle\right]\right| \lesssim\left(\|v\|_{L^{\infty}}+\|v\|_{L^{2}}\right)\|v\|_{L^{2}}\||A|\|_{L^{2} \rightarrow L^{2}} .
$$

The claim for small $t$ also follows from these bounds without integrating by parts.

Now we consider $\Gamma_{2}$. By the absolute boundedness of $A$ and Lemma 5.2, we have

$$
A v R_{1} g=\widetilde{g}_{1}+\widetilde{g}_{2} \widetilde{O}_{1}(z)
$$

for some $\widetilde{g}_{1}, \widetilde{g}_{2} \in L^{2}$. Using this we write the oscillatory integral as

$$
\int_{\mathbb{R}^{4}} f(y) v\left(x_{1}\right) \int_{0}^{\infty} e^{i t \sqrt{z^{2}+m^{2}} \pm i z\left|x_{1}-y\right|} a\left(z, y, x_{1}\right) d z d x_{1} d y
$$

where

$$
a\left(z, y, x_{1}\right)=\left(\widetilde{g}_{1}\left(x_{1}\right)+\widetilde{g}_{2}\left(x_{1}\right) \widetilde{O}_{1}(z)\right) \frac{z \chi(z)}{\sqrt{z^{2}+m^{2}}} \widetilde{\chi}\left(z\left|y-x_{1}\right|\right) \widetilde{\omega}_{ \pm}\left(z\left(y-x_{1}\right)\right) .
$$

Therefore, using Lemma 3.4, we bound the integral above by

$$
\frac{1}{\langle t\rangle} \int_{\mathbb{R}^{4}}|f(y)|\left|v\left(x_{1}\right)\right|\left(\left|\widetilde{g}_{1}\left(x_{1}\right)\right|+\left|\widetilde{g}_{2}\left(x_{1}\right)\right|\right) d x_{1} d y \leq \frac{1}{\langle t\rangle}\|f\|_{L^{1}}\|v\|_{L^{2}}\left(\left\|\widetilde{g}_{1}\right\|_{L^{2}}+\left\|\widetilde{g}_{2}\right\|_{L^{2}}\right) .
$$

Finally we consider $\Gamma_{3}$. Using $\mathcal{R}_{0}=\mathcal{R}_{L}+\mathcal{R}_{H}$, we bound the contribution of $\mathcal{R}_{H}$ to the integral by (with $r=\left|x-x_{1}\right|, s=\left|y-y_{1}\right|$ )

$$
\sup _{r, s}\left|\int_{0}^{\infty} e^{i t \sqrt{z^{2}+m^{2}} \pm i z(r+s)} \frac{z \chi(z)}{\sqrt{z^{2}+m^{2}}} \widetilde{\chi}(z r) \omega_{1}^{ \pm}(z r) \widetilde{\chi}(z s) \widetilde{\omega}_{ \pm}(z s) d z\right|,
$$

which is $O(1 /\langle t\rangle)$ by Lemma 3.4 noting that

$$
\omega_{1}^{ \pm}(z r) \widetilde{\omega}_{ \pm}(z s)=\widetilde{O}_{1}\left((1+z(r+s))^{-1 / 2}\right) .
$$

For $\mathcal{R}_{L}$ we note that

$$
z \mathcal{R}_{L}=z R_{1}+\widetilde{O}_{1}\left(z^{1-}\left(1+\log ^{-}(|x-y|)\right)\right.
$$


Therefore, since $\omega_{1}^{ \pm}$behaves like $z \omega_{ \pm}$, the argument above for $\Gamma_{2}$ takes care of the first summand. For the second summand, writing $k\left(y_{1}, y\right)=\left(1+\log ^{-}\left(y_{1}-y\right)\right)$ and using Lemma 3.4 we bound its contribution by

$$
\begin{aligned}
\frac{1}{\langle t\rangle} \int_{\mathbb{R}^{8}}\left|f(y)\left\|v^{*}\left(x_{1}\right)\right\| A\left(x_{1}, y_{1}\right) \| v\left(y_{1}\right)\right| k\left(y_{1}-y\right)|g(x)| d x_{1} d y_{1} d x d y & \\
& \lesssim \frac{1}{\langle t\rangle} \sup _{y}\left\|v(\cdot)\left(1+\log ^{-}(\cdot-y)\right)\right\|_{L^{2}}\||A|\|_{L^{2} \rightarrow L^{2}}\|v\|_{L^{2}}
\end{aligned}
$$

Now we consider the contribution of the term $h_{ \pm}(z)^{-1} S$ from (38) (the contribution of $h_{ \pm}(z)^{-1} S S_{1} D_{1} S_{1} S$ is handled similarly). By symmetry and the discussion above, it suffices to consider the terms

$$
\Gamma_{1}:=R_{1} v^{*} S v\left(\frac{\mathcal{R}_{L}^{+}}{h_{+}(z)}-\frac{\mathcal{R}_{L}^{-}}{h_{-}(z)}\right), \quad \Gamma_{2}:=h_{+}^{-1} R_{1} v^{*} S v \mathcal{R}_{H}^{+}, \quad \Gamma_{3}:=h_{+}^{-1} R_{4}^{+} v^{*} S v \mathcal{R}_{0}^{+} .
$$

Proposition 5.4. The assertion of Proposition 5.3 is valid for each $\Gamma_{j}$ defined in (51).

Proof. The proof for $\Gamma_{2}$ and $\Gamma_{3}$ follows from Proposition 5.3 by noting that $h_{ \pm}^{-1}=\widetilde{O}_{1}(1)$. For $\Gamma_{1}$, it suffices to obtain the inequality (49). Note that using Lemma 2.2, the identity $h^{ \pm}(z)=c_{1}+g^{ \pm}(z)+c_{2}$, and

$$
h^{+}(z)^{-1}-h^{-}(z)^{-1}=\widetilde{O}_{1}\left(\log ^{-2} z\right)
$$

we have

$$
\begin{aligned}
& \left(\frac{\mathcal{R}_{L}^{+}}{h_{+}(z)}-\frac{\mathcal{R}_{L}^{-}}{h_{-}(z)}\right)=\chi(z|x-y|)\left(R_{1}+c M_{11}\right) \widetilde{O}_{1}\left(\log ^{-2} z\right) \\
& \quad+\chi(z|x-y|)\left[-\frac{m I_{1}}{\pi} \log (|x-y|) \widetilde{O}_{1}\left(\log ^{-2} z\right)+\widetilde{O}_{1}\left(z^{3 / 2}\left(|x-y|^{3 / 2}+\log ^{-}|x-y|\right)\right)\right] .
\end{aligned}
$$

The contribution of the first term is $O\left(\langle t\rangle^{-1}\right)$ as in the proof of Proposition 5.3 using (42) for $f$ and $g$. Using (50), the contribution of the the second term to the left hand side of (49) can be bounded by

$$
\begin{aligned}
\int_{\mathbb{R}^{4}} \mid v\left(y_{1}\right) & || f(y)\left|\int_{0}^{\infty}\right| \partial_{z}\left[\left(\widetilde{g}_{1}\left(y_{1}\right)+\widetilde{g}_{2}\left(y_{1}\right) \widetilde{O}_{1}(z)\right) \chi(z)\right. \\
& \left.\chi\left(z\left|y-y_{1}\right|\right) \log \left(\left|y-y_{1}\right|\right) \widetilde{O}_{1}\left(\log ^{-2} z\right)\right] \mid d z d y_{1} d y \\
\lesssim & \int_{\mathbb{R}^{4}}\left|v\left(y_{1}\right)\right||f(y)|\left(\left|\widetilde{g}_{1}\left(y_{1}\right)\right|+\left|\widetilde{g}_{2}\left(y_{1}\right)\right|\right) \\
& \int_{0}^{\infty} \chi(z) \log \left(\left|y-y_{1}\right|\right)\left(\frac{\chi\left(z\left|y-y_{1}\right|\right)}{z \log ^{3}(z)}+\frac{\left|y-y_{1}\right| \chi^{\prime}\left(z\left|y-y_{1}\right|\right)}{\log ^{2}(z)}\right) d z d y_{1} d y \\
\lesssim & \left\|v\left(y_{1}\right) \int_{0}^{\infty} \chi(z)\left(1+\log ^{-}\left(\left|y-y_{1}\right|\right)\right)\left(\frac{\chi\left(z\left|y-y_{1}\right|\right)}{z \log ^{2}(z)}+\left|y-y_{1}\right| \chi^{\prime}\left(z\left|y-y_{1}\right|\right)\right) d z\right\|_{L_{y^{\infty}} L_{y_{1}}^{2}}
\end{aligned}
$$




$$
\lesssim\left\|v\left(y_{1}\right)\left(1+\log ^{-}\left(\left|y-y_{1}\right|\right)\right)\right\|_{L_{y}^{\infty} L_{y_{1}}^{2}} \lesssim 1 .
$$

In the second to last inequality, we used that $|\log z|^{-1} \lesssim 1$ and

$$
\left|\chi(z) \chi\left(z\left|y-y_{1}\right|\right) \log \right| y-y_{1}|| \lesssim 1+\log ^{-}\left|y-y_{1}\right|+|\log z| .
$$

The contribution of the last term can be handled similarly.

Now we consider the contribution of the error term $E^{ \pm}(z)$ from (38). By symmetry and the discussion above, and dropping \pm indices, it suffices to consider the terms

$$
\Gamma_{1}:=R_{1} v^{*} E v \mathcal{R}_{L}, \quad \Gamma_{2}:=R_{1} v^{*} E v \mathcal{R}_{H}, \quad \Gamma_{3}:=R_{4} v^{*} E v \mathcal{R}_{0} .
$$

Proposition 5.5. The assertion of Proposition 5.3 is valid for each $\Gamma_{j}$ defined in (52).

Proof. For $\Gamma_{1}$, as in the proof of Proposition 5.3 it suffices to prove that

$$
\left\|\partial_{z}\left[\chi(z)\left\langle\Gamma_{1} f, g\right\rangle\right]\right\|_{L_{z}^{1}} \lesssim 1
$$

Note that

$$
\partial_{z}\left(R_{1} v^{*} E v \mathcal{R}_{L}\right)=\left(\partial_{z} R_{1}\right) v^{*} E v \mathcal{R}_{L}+R_{1} v^{*}\left(\partial_{z} E\right) v \mathcal{R}_{L}+R_{1} v^{*} E v\left(\partial_{z} \mathcal{R}_{L}\right) .
$$

We only consider the contribution of the last summand, the others are similar. We have

$$
\partial_{z} \mathcal{R}_{L}=\partial_{z} R_{1}+r \chi^{\prime}(z r) \log (z r)+O(1 / z)=O\left(\frac{1}{r^{0+} z^{1+}}\right) .
$$

We write (with $\left.\widetilde{E}\left(x_{1}, y_{1}\right)=\sup _{z} z^{-1 / 2}\left|E\left(z, x_{1}, y_{1}\right)\right|\right)$

$$
\begin{aligned}
\int \chi(z)\left|\left\langle R_{1} v^{*} E v\left(\partial_{z} \mathcal{R}_{L}\right) f, g\right\rangle\right| & d z \\
& \lesssim \int \frac{\chi(z)}{z^{\frac{1}{2}+}}\left|\left(v R_{1} g\right)\left(z, x_{1}\right)\right|\left|\widetilde{E}\left(x_{1}, y_{1}\right)\right| \frac{\left|v\left(y_{1}\right)\right|}{\left|y-y_{1}\right|^{0+}}|f(y)| d x_{1} d y_{1} d y d z .
\end{aligned}
$$

By Cauchy-Schwarz and Lemma [5.2, we estimate the $x_{1}$ integral by

$$
\left\|\left(v R_{1} g\right)\left(z, x_{1}\right)\right\|_{L_{x_{1}}^{2}}\left\|\widetilde{E}\left(x_{1}, y_{1}\right)\right\|_{L_{x_{1}}^{2}} \lesssim\left\|\widetilde{E}\left(x_{1}, y_{1}\right)\right\|_{L_{x_{1}}^{2}},
$$

uniformly in $z$. Therefore, we estimate the integral above by

$$
\int\left\|\widetilde{E}\left(x_{1}, y_{1}\right)\right\|_{L_{x_{1}}^{2}} \frac{\left|v\left(y_{1}\right)\right|}{\left|y-y_{1}\right|^{0+}}|f(y)| d y_{1} d y \lesssim\|f\|_{L^{1}}\|\widetilde{E}\|_{H S} \sup _{y}\left\|v /|y-\cdot|^{0+}\right\|_{L^{2}} \lesssim 1,
$$

where we used Cauchy-Schwarz in the $y_{1}$ integral.

The bound for the contribution of the first summand on the right hand side of (53) is nearly identical. For the second summand, one must use Lemma 5.2 twice and use that $\sup _{z}\left|z^{\frac{1}{2}} \partial_{z} E\left(z, x_{1}, y_{1}\right)\right|$ is Hilbert-Schmidt.

We now consider $\Gamma_{2}$, whose contribution to the Stone formula is given by

$$
\int_{\mathbb{R}^{4}} \int_{0}^{\infty} e^{i t \sqrt{z^{2}+m^{2}} \pm i\left|y-y_{1}\right|} \frac{z \chi(z)}{\sqrt{z^{2}+m^{2}}}\left[v R_{1} g\right]\left(z, x_{1}\right) E\left(z, x_{1}, y_{1}\right)
$$




$$
v\left(y_{1}\right) \widetilde{\omega}_{ \pm}\left(z\left(y_{1}-y\right)\right) f(y) d x_{1} d y_{1} d y d z .
$$

We apply Lemma 3.4 to this integral with

$$
a(z)=\frac{z \chi(z)}{\sqrt{z^{2}+m^{2}}} v R_{1} g E v \widetilde{\omega}_{ \pm}\left(z\left(y_{1}-y\right)\right)
$$

we note that by Lemma 5.2 we have

$$
v R_{1} g\left(z, x_{1}\right)=g_{1}\left(x_{1}\right)+g_{2}\left(x_{1}\right) \widetilde{O}_{1}(z) .
$$

for some $g_{1}, g_{2} \in L^{2}$. This along with the bounds on $E$ from Lemma 4.5 and the definition of $\widetilde{\omega}_{ \pm}$yields the bound

$$
\begin{aligned}
|a(z)| & \lesssim\left(\left|g_{1}\left(x_{1}\right)\right|+\left|g_{2}\left(x_{1}\right)\right|\right) \sup _{z}\left|z^{-\frac{1}{2}} E\left(z, x_{1}, y_{1}\right)\right|\left|v\left(y_{1}\right)\right| \frac{z \chi(z) \widetilde{\chi}\left(z\left|y-y_{1}\right|\right)}{\left(1+z\left|y-y_{1}\right|\right)^{\frac{1}{2}}} \\
\left|\partial_{z} a(z)\right| & \lesssim\left|g_{2}\left(x_{1}\right)\right|\left(\sup _{z}\left|z^{-\frac{1}{2}} E\left(z, x_{1}, y_{1}\right)\right|+\sup _{z}\left|z^{\frac{1}{2}} \partial_{z} E\left(z, x_{1}, y_{1}\right)\right|\right)\left|v\left(y_{1}\right)\right| \frac{\chi(z) \widetilde{\chi}\left(\left|y-y_{1}\right|\right)}{\left(1+z\left|y-y_{1}\right|\right)^{\frac{1}{2}}}
\end{aligned}
$$

This implies the desired time decay bound using Lemma 3.4. The spatial integrals can be controlled as in the case of $\Gamma_{1}$.

For $\Gamma_{3}$, writing $\mathcal{R}_{0}=\mathcal{R}_{L}+\mathcal{R}_{H}$, the contribution of $\mathcal{R}_{L}$ follows as in the bounds of $\Gamma_{2}$. For $\mathcal{R}_{H}$, let $r=\left|x-x_{1}\right|+\left|y-y_{1}\right|$ and

$$
a(z)=\frac{z \chi(z) E\left(x_{1}, y_{1}, z\right)}{h(z) \sqrt{z^{2}+m^{2}}} \widetilde{\chi}\left(z\left|y-y_{1}\right|\right) \widetilde{\chi}\left(z\left|x-x_{1}\right|\right) \widetilde{\omega}^{ \pm}\left(z\left|x-x_{1}\right|\right) \widetilde{\omega}^{ \pm}\left(z\left|y-y_{1}\right|\right) .
$$

Note that $a(z)$ satisfies the bounds

$$
\begin{aligned}
|a(z)| & \lesssim \frac{z \chi(z) \widetilde{\chi}(z r)}{(1+z r)^{1 / 2}} \sup _{z}\left|E\left(x_{1}, y_{1}, z\right)\right|, \\
\left|\partial_{z} a(z)\right| & \lesssim \frac{\chi(z) \widetilde{\chi}(z r)}{(1+z r)^{1 / 2}} \sup _{z}\left(\left|E\left(x_{1}, y_{1}, z\right)\right|+\left|z \partial_{z} E\left(x_{1}, y_{1}, z\right)\right|\right) .
\end{aligned}
$$

Therefore, using Lemma 5.2 and then the bounds for $E$ and $\partial_{z} E$ given in Lemma 4.5 , we obtain the bound

$$
\frac{1}{\langle t\rangle} \int_{\mathbb{R}^{8}} \sup _{z}\left(\left|E\left(x_{1}, y_{1}, z\right)\right|+\left|z \partial_{z} \mathcal{E}\left(x_{1}, y_{1}, z\right)\right|\right)\left|v\left(x_{1}\right)\left\|v\left(y_{1}\right)|| f(x)\right\| g(y)\right| d x d y d x_{1} d y_{1} \lesssim \frac{1}{\langle t\rangle} .
$$

To control the 's-wave' term with $h^{ \pm} S_{1} D_{1} S_{1}$ on the right hand side of (38). In particular, we need to consider terms of the form

$$
\begin{aligned}
& \Gamma_{1}=\left[h^{+}(z)-h^{-}(z)\right] R_{1} v^{*} S_{1} D_{1} S_{1} v \mathcal{R}_{0}^{ \pm} \\
& \Gamma_{2}=h^{+}(z) R_{1} v^{*} S_{1} D_{1} S_{1} v\left[\mathcal{R}_{0}^{+}-\mathcal{R}_{0}^{-}\right](z), \quad \Gamma_{3}=h^{+}(z) R_{4}^{+} v^{*} S_{1} D_{1} S_{1} v \mathcal{R}_{0}^{+} .
\end{aligned}
$$

Proposition 5.6. The assertion of Proposition 5.3 is valid for each $\Gamma_{j}$ defined in (54). 
Proof. For $\Gamma_{1}$, note that $h^{+}(z)-h^{-}(z)=c$. Recalling Proposition 5.3 for $\Gamma_{2}$ defined in (48), it suffices to consider the contribution of

$$
R_{1} v^{*} S_{1} D_{1} S_{1} v \mathcal{R}_{L}^{+}
$$

Using (22), we write

$$
\mathcal{R}_{L}^{+}(z)(x, y)=R_{1}-\frac{m I_{1}}{\pi} \log (z|x-y|) \chi(z|x-y|)+\widetilde{O}_{1}\left(z^{3 / 2}\left(|x-y|^{3 / 2}+\log ^{-}|x-y|\right)\right),
$$

we note that the contribution of the third summand follows from the analysis of $\Gamma_{1}$ in Proposition 5.4. The contribution of the first summand is easier using Lemma 5.2 for both $f$ and $g$. For the contribution of the second summand we need to use the orthogonality $S_{1} v M_{11}=0$, which holds since $S_{1} \leq Q$. Let

$$
F\left(z, y, y_{1}\right)=-\frac{m I_{1}}{\pi} \log \left(z\left|y_{1}-y\right|\right) \chi\left(z\left|y_{1}-y\right|\right)+\frac{m I_{1}}{\pi} \log (z\langle y\rangle) \chi(z\langle y\rangle) .
$$

By the orthogonality $S_{1} v M_{11}=0$, and using Lemma 5.2, the contribution of the second summand to the left hand side of (49) is given by

$$
\left\|\partial_{z}\left[\chi(z) \int_{\mathbb{R}^{6}}\left[S_{1} D_{1} S_{1}\right]\left(x_{1}, y_{1}\right) v\left(y_{1}\right) F\left(z, y_{1}, y\right) f(y)\left[g_{1}\left(x_{1}\right)+g_{2}\left(x_{1}\right) \widetilde{O}_{1}(z)\right]\left(x_{1}\right) d x_{1} d y_{1} d y\right]\right\|_{L_{z}^{1}},
$$

where $\left\|g_{j}\right\|_{L^{2}} \lesssim 1$. We have the following bounds for $z \lesssim z_{0}$ (see [41], [23, Lemma 3.3])

$$
\left|F\left(z, y_{1}, y\right)\right| \lesssim \int_{0}^{z_{0}}\left|\partial_{z} F\left(z, y_{1}, y\right)\right| d z+\left|F\left(0+, y_{1}, y\right)\right| \lesssim k_{2}\left(y_{1}, y\right)
$$

where $k_{2}\left(y_{1}, y\right):=1+\log ^{+}\left(\left|y_{1}\right|\right)+\log ^{-}\left(\left|y-y_{1}\right|\right)$. Therefore, we can estimate the integral above by

$$
\begin{aligned}
\int_{\mathbb{R}^{6}}\left|\left[S_{1} D_{1} S_{1}\right]\left(x_{1}, y_{1}\right)\left\|v\left(y_{1}\right) k_{2}\left(y_{1}, y\right)\right\| f(y)\right|\left(\left|g_{1}\left(x_{1}\right)\right|+\left|g_{2}\left(x_{1}\right)\right|\right) d x_{1} d y_{1} d y \\
\leq\left[\sup _{y}\left\|v(\cdot) k_{2}(\cdot, y)\right\|_{L^{2}}\right]\left\|\left|S_{1} D_{1} S_{1}\right|\right\|_{L^{2} \rightarrow L^{2}}\left\|\left|g_{1}\left(x_{1}\right)\right|+\left|g_{2}\left(x_{1}\right)\right|\right\| L_{L^{2}}\|f\|_{L^{1}} \lesssim 1 .
\end{aligned}
$$

The contribution of $\Gamma_{3}$ can be handled as in Proposition 5.3 since the additional $z$ factor in $R_{4}$ kills the logarithm coming from $h(z)$.

For the contribution of $\Gamma_{2}$, we write

$$
\begin{aligned}
{\left[\mathcal{R}_{0}^{+}-\mathcal{R}_{0}^{-}\right](z)(x, y)=\left[-i \alpha \cdot \nabla+m \beta+\sqrt{m^{2}+z^{2}} I\right] J_{0}(z|x-y|) } \\
=2 m I_{1} J_{0}+\left(R_{4}^{+}-R_{4}^{-}\right)+\widetilde{O}_{1}(z) \chi(z|x-y|) J_{0} .
\end{aligned}
$$

The contribution of the last two summand is similar to the cases above. The contribution of the first summand can be handled using the orthogonality property as above and as in [23]; the functions $G, \widetilde{G}$ from [23] which have an additional factor of $z$ replace the function $F$ above. The rest of the analysis is identical to the one above for low energies and to the analysis of the terms containing $R_{4}$ for the high energies. 
We are now ready to prove Theorem 5.1 .

Proof of Theorem 5.1. Using the expansion for the perturbed resolvents given in (38). The first term is controlled by the bounds for the evolution of the free resolvent in Theorem 3.1 , specifically (25). Propositions 5.3, 5.4, 5.5 control the contribution of the operators $A, S-S S_{1} D_{1} S_{1} S$ and $E^{ \pm}(z)$ respectively. This establishes the theorem in the case when the threshold $\lambda=m$ is regular. If there is a resonance of the first kind, that is an s-wave resonance at $\lambda=m$, we bound the additional $h_{ \pm}(z) S_{1} D_{1} S_{1}$ term with Proposition 5.6 .

\section{High ENERGy Dispersive EStimates}

We now seek to bound the perturbed Dirac evolution at energies separated from the threshold. In particular, we show

Proposition 6.1. Under Assumption 1.1, the following bound holds for any $H^{1} \times H^{1}\left(\mathbb{R}^{2}\right)$ atoms $f$ and $g$.

$$
\left|\int_{0}^{\infty} e^{-i t \sqrt{z^{2}+m^{2}}} \frac{z}{\sqrt{z^{2}+m^{2}}} \chi_{j}(z)\left\langle\left[\mathcal{R}_{V}^{+}(\lambda)-\mathcal{R}_{V}^{-}(\lambda)\right] f, g\right\rangle d z\right| \lesssim \min \left(2^{2 j}, 2^{7 j / 2}|t|^{-1}\right) .
$$

provided the components of $V$ satisfy the bound $\left|V_{i j}(x)\right| \lesssim\langle x\rangle^{-2-}$.

As in the low-energy part of the evolution, we use the Hardy space $H^{1}$ in place of the Lebesgue space $L^{1}$. One can prove such bounds with $L^{1}$, though it requires further iteration of the Born series, which requires more complicated computations and loss of more derivatives on the initial data than presented here.

The following lemma from [22] will be useful to control the spatial integrals that arise in our frequency-localized bounds.

Lemma 6.2. Fix $u_{1}, u_{2} \in \mathbb{R}^{n}$ and let $0 \leq k, \ell<n, \beta>0, k+\ell+\beta \geq n, k+\ell \neq n$. We have

$$
\int_{\mathbb{R}^{n}} \frac{\left\langle z_{1}\right\rangle^{-\beta-}}{\left|z_{1}-u_{1}\right|^{k}\left|z_{1}-u_{2}\right|^{\ell}} d z_{1} \lesssim \begin{cases}\left(\frac{1}{\left|u_{1}-u_{2}\right|}\right)^{\max (0, k+\ell-n)} & \left|u_{1}-u_{2}\right| \leq 1 \\ \left(\frac{1}{\left|u_{1}-u_{2}\right|}\right)^{\min (k, \ell, k+\ell+\beta-n)} & \left|u_{1}-u_{2}\right|>1 .\end{cases}
$$

We begin by employing the resolvent expansion

$$
\mathcal{R}_{V}^{ \pm}(\lambda)=\mathcal{R}_{0}^{ \pm}(\lambda)-\mathcal{R}_{0}^{ \pm}(\lambda) V \mathcal{R}_{0}^{ \pm}(\lambda)+\mathcal{R}_{0}^{ \pm}(\lambda) V \mathcal{R}_{V}^{ \pm}(\lambda) V \mathcal{R}_{0}^{ \pm}(\lambda) .
$$

We already discussed the required bounds for the contribution of the free resolvent in Theorem 3.1. We now consider the contribution of the second term in (57). Using the estimates in the previous sections, see (41), (39), and the discussion preceding Lemma [5.2, we have (for $\left.z \gtrsim 1, \lambda=\sqrt{z^{2}+m^{2}}\right)$

$$
\begin{aligned}
& \mathcal{R}_{L}^{ \pm}(z)(x, y)=\chi(z|x-y|)\left(\frac{i \alpha \cdot(x-y)}{2 \pi|x-y|^{2}}+\widetilde{O}_{1}\left(z(z|x-y|)^{0-}\right)\right), \\
& \mathcal{R}_{H}^{ \pm}(z)(x, y)=e^{ \pm i z|x-y|} \widetilde{w}_{ \pm}(z|x-y|),
\end{aligned}
$$




$$
\frac{\mathcal{R}_{L}^{+}(z)(x, y)-\mathcal{R}_{L}^{-}(z)(x, y)}{\sqrt{z^{2}+m^{2}}}=\widetilde{O}_{1}\left(z / \sqrt{z^{2}+m^{2}}\right) .
$$

By symmetry, it suffices to consider the contributions of

$$
\Gamma_{1}:=\left(\mathcal{R}_{L}^{+}-\mathcal{R}_{L}^{-}\right) V \mathcal{R}_{L}^{+}, \quad \Gamma_{2}:=\mathcal{R}_{L}^{+} V \mathcal{R}_{H}^{+}, \quad \Gamma_{3}:=\mathcal{R}_{H}^{+} V \mathcal{R}_{H}^{+}
$$

to the Stone's formula (56).

Lemma 6.3. The following bound holds for each $k=1,2,3$

$$
\left|\int_{0}^{\infty} e^{-i t \sqrt{z^{2}+m^{2}}} \frac{z}{\sqrt{z^{2}+m^{2}}} \chi_{j}(z) \Gamma_{k} d z\right| \lesssim \min \left(2^{2 j}, 2^{2 j}|t|^{-1 / 2}, 2^{5 j / 2}|t|^{-1}\right)
$$

provided the components of $V$ satisfy the bound $\left|V_{i j}(x)\right| \lesssim\langle x\rangle^{-2-}$.

Proof. For $\Gamma_{1}$, we need to consider an integral which can be written as

$$
\left|\int_{\mathbb{R}^{2}} \int_{0}^{\infty} e^{-i t \sqrt{z^{2}+m^{2}}} \chi_{j}(z) \chi(z|x-u|) V(u) \chi(z|u-y|) \widetilde{O}_{1}(z /|u-y|) d z d u\right| .
$$

We can bound the integral by

$$
\min \left(2^{2 j}, 2^{j} / t\right) \int_{\mathbb{R}^{2}} \frac{|V(u)|}{|u-y|} d u \lesssim \min \left(2^{2 j}, 2^{j} / t\right)
$$

To obtain this we estimated the $z$ integral by ignoring the phase, and by an integration by parts as before. The $u$ integral is clearly bounded by Lemma 6.2.

For $\Gamma_{2}$ and $\Gamma_{3}$, we note that direct integration implies the bound $2^{2 j}$ as above. To obtain time decay we employ Lemma 3.5 to the oscillatory integral that the phase(s) in $\mathcal{R}_{H}(z)$ provide. We estimate $\Gamma_{3}$ only, $\Gamma_{2}$ is bounded similarly with a smaller power of $2^{j}$. With $\phi_{ \pm}(z)=$ $\sqrt{z^{2}+m^{2}} \mp z(|x-u|+|u-y|) / t$, we consider

$$
\int_{\mathbb{R}^{2}} \int_{0}^{\infty} e^{-i t \phi_{ \pm}(z)} \chi_{j}(z) \widetilde{O}_{1}\left(z^{2}\right) \widetilde{\omega}_{+}(z|x-u|) \widetilde{\omega}_{+}(z|u-y|) d z d u
$$

Define $r:=\max (|x-u|,|u-y|)$ and $s:=\min (|x-u|,|u-y|)$, we then rewrite the integrand as

$$
e^{-i t \phi_{+}(z)} \frac{z \chi_{j}(z) \widetilde{\chi}(z r)}{(1+z r)^{\frac{1}{2}}} \frac{z^{\frac{1}{2}}}{s^{\frac{1}{2}}}
$$

Since $r \approx r+s$, and $z^{\frac{1}{2}} \approx 2^{j / 2}$, we apply Lemma 3.5 to bound the $z$ integral with

$$
2^{j / 2} \min \left(2^{2 j}, 2^{\frac{3 j}{2}}|t|^{-1 / 2}, 2^{2 j}|t|^{-1}\right) \int_{\mathbb{R}^{2}} \frac{|V(u)|}{|u-y|^{\frac{1}{2}}} d u
$$

Here, without loss of generality, we took $s=|u-y|$. The $u$ integral is bounded by the decay of $V$ and Lemma 6.2. The case of $k=2$ varies only in that the final integrand is bounded by

$$
\min \left(2^{2 j}, 2^{\frac{3 j}{2}}|t|^{-1 / 2}, 2^{2 j}|t|^{-1}\right) \int_{\mathbb{R}^{2}} \frac{|V(u)|}{|u-y|} d u .
$$

The following lemma finishes the proof of Proposition 6.1 . 
Lemma 6.4. The following bound holds for any $H^{1} \times H^{1}\left(\mathbb{R}^{2}\right)$ atoms $f$ and $g$.

$$
\left|\int_{0}^{\infty} e^{-i t \sqrt{z^{2}+m^{2}}} \frac{z \chi_{j}(z)}{\sqrt{z^{2}+m^{2}}}\left\langle\mathcal{R}_{0}^{ \pm}(\lambda) V \mathcal{R}_{V}^{ \pm}(\lambda) V \mathcal{R}_{0}^{ \pm}(\lambda) f, g\right\rangle d z\right| \lesssim \min \left(2^{2 j}, 2^{7 j / 2}|t|^{-1}\right) .
$$

provided the components of $V$ satisfy the bound $\left|V_{i j}(x)\right| \lesssim\langle x\rangle^{-2-}$.

Proof. In this proof we consider only the case $t>0$, and the ' + ' terms, and drop the superscripts. By symmetry, it suffices to consider the contributions of the following to (60):

$$
\Gamma_{1}:=\mathcal{R}_{L} V \mathcal{R}_{V} V \mathcal{R}_{L}, \quad \Gamma_{2}:=\mathcal{R}_{L} V \mathcal{R}_{V} V \mathcal{R}_{H}, \quad \Gamma_{3}:=\mathcal{R}_{H} V \mathcal{R}_{V} V \mathcal{R}_{H}
$$

Consider the contribution of $\Gamma_{1}$. We rewrite $\mathcal{R}_{L}$, see (58), as follows:

$$
\begin{aligned}
\mathcal{R}_{L}(z)(x, y) & =\frac{i \alpha \cdot(x-y)}{2 \pi|x-y|^{2}}+\widetilde{\chi}(z|x-y|) \widetilde{O}\left(|x-y|^{-1}\right)+\chi(z|x-y|) \widetilde{O}_{1}\left(z(z|x-y|)^{0-}\right) \\
& =\frac{i \alpha \cdot(x-y)}{2 \pi|x-y|^{2}}+\widetilde{O}_{1}\left(z^{1 / 2}|x-y|^{-1 / 2}\right)=: \frac{i \alpha \cdot(x-y)}{2 \pi|x-y|^{2}}+\mathcal{R}_{L 1} .
\end{aligned}
$$

Using Theorem 1 in [20], the first summand above maps $H^{1}$ to $L^{2}$. Thus, we can write

$$
\left\langle\Gamma_{1} f, g\right\rangle=\left\langle\mathcal{R}_{V} V \tilde{f}, V \widetilde{g}\right\rangle+\left\langle\mathcal{R}_{V} V \mathcal{R}_{L 1} f, V \widetilde{g}\right\rangle+\left\langle V \tilde{f}, \mathcal{R}_{V} V \mathcal{R}_{L 1} g\right\rangle+\left\langle\mathcal{R}_{L 1} V \mathcal{R}_{V} V \mathcal{R}_{L 1} f, g\right\rangle,
$$

where $\widetilde{f}, \widetilde{g} \in L^{2} \times L^{2}$. Therefore, by limiting absorption principle we have:

$$
\begin{aligned}
\left|\left\langle\Gamma_{1} f, g\right\rangle\right| \lesssim\|V \widetilde{g}\|_{L_{\sigma}^{2}}\|V \widetilde{f}\|_{L_{\sigma}^{2}}+z^{1 / 2}\|V \widetilde{g}\|_{L_{\sigma}^{2}}\|f\|_{L^{1}} \sup _{u}\left\|\frac{V(\cdot)}{|\cdot-u|^{1 / 2}}\right\|_{L_{\sigma}^{2}} \\
\quad+z^{1 / 2}\|V \widetilde{f}\|_{L_{\sigma}^{2}}\|g\|_{L^{1}} \sup _{u}\left\|\frac{V(\cdot)}{|\cdot-u|^{1 / 2}}\right\|_{L_{\sigma}^{2}}+z\|f\|_{L^{1}}\|g\|_{L^{1}} \sup _{u}\left\|\frac{V(\cdot)}{|\cdot-u|^{1 / 2}}\right\|_{L_{\sigma}^{2}}^{2} \lesssim z .
\end{aligned}
$$

Using this bound we estimate the contribution of $\Gamma_{1}$ to (60) by $2^{2 j}$. The same bound holds for $\Gamma_{2}$ and $\Gamma_{3}$. We also have

$$
\left|\partial_{z}\left\langle\Gamma_{1} f, g\right\rangle\right| \lesssim z
$$

since the worst terms are the ones when the derivative hits $\mathcal{R}_{V}$. Using this bound after an integration by parts we estimate the contribution of $\Gamma_{1}$ to (60) by $2^{2 j} / t$.

For $\Gamma_{3}$, it suffices to estimate

$$
\sup _{x, y}\left|\int_{0}^{\infty} e^{-i 2^{-3 j} t \phi(z)} a(z, x, y) d z\right|
$$

where

$$
\phi(z)=2^{3 j}\left(\sqrt{z^{2}+m^{2}}-z(|x|+|y|) / t\right),
$$

and (with $r=\left|x-u_{1}\right|, s=\left|u_{1}-y\right|$ )

$$
a(z, x, y)=\int_{\mathbb{R}^{4}} \chi_{j}(z) \widetilde{O}_{1}\left(z^{2}\right) e^{i z(r-|x|)} \widetilde{\omega}(z r)\left[V \mathcal{R}_{V} V\right]\left(u_{1}, u_{2}\right) e^{i z(s-|y|)} \widetilde{\omega}(z s) d u_{1} d u_{2} .
$$


Note that in the support of $a$, we have $\phi^{\prime \prime} \approx 1$. Also note that, using Lemma 6.2 and the limiting absorption principle, we have

$$
|a(z, x, y)|+\left|\partial_{z} a(z, x, y)\right| \lesssim 2^{j} \chi_{j}(z)\langle x\rangle^{-1 / 2}\langle y\rangle^{-1 / 2} .
$$

Therefore by Lemma 3.3, we estimate the integral above by

$$
\int_{\left|z-z_{0}\right|<\sqrt{2^{3 j} / t}}|a(z)| d z+t^{-1} 2^{3 j} \int_{\left|z-z_{0}\right|>\sqrt{2^{3 j} / t}}\left(\frac{|a(z)|}{\left|z-z_{0}\right|^{2}}+\frac{\left|a^{\prime}(z)\right|}{\left|z-z_{0}\right|}\right) d z
$$

where $z_{0}=m \frac{|x|+|y|}{\sqrt{t^{2}-(|x|+|y|)^{2}}}$. In the case when $z_{0}$ is in a small neighborhood of the support of $a$ we must have $t \approx|x|+|y|$. Therefore, in this case, we have the bound

$$
2^{j}\langle x\rangle^{-1 / 2}\langle y\rangle^{-1 / 2}\left(\sqrt{2^{3 j} / t}+t^{-1} 2^{3 j} \frac{2^{j}}{\sqrt{2^{3 j} / t}}\right) \lesssim 2^{7 j / 2} / t .
$$

In the case $t \not \approx|x|+|y|$, we have

$$
\left|\partial_{z}\left(\sqrt{z^{2}+m^{2}}-z(|x|+|y|) / t\right)\right| \gtrsim 1
$$

An integration by parts together with the bounds on $a$ imply that the integral is bounded by $2^{2 j} / t$. The proof for $\Gamma_{2}$ is similar to the cases considered above.

\section{Classification of threshold Resonances}

In this section we provide a full characterization of threshold obstructions. We classify distributional solutions to $H \psi=m \psi$ and relate them to the spectral subspaces and terms that arise in the inversion of the operators $M^{ \pm}(z)=U+v \mathcal{R}_{0}^{ \pm}\left(\sqrt{m^{2}+z^{2}}\right) v^{*}, \quad 0<z \ll 1$. We start with the absolute boundedness of the operator $Q D_{0} Q$ in the case $S_{1}=0$. In the case $S_{1} \neq 0$, the proof is similar.

Lemma 7.1. If $\left|v_{i j}(x)\right| \lesssim\langle x\rangle^{-1-}$ then $Q D_{0} Q$ is an absolutely bounded operator.

Proof. This is similar to the proof Lemma 8 in [41]. Assume that $0 \neq f \in Q\left(L^{2} \times L^{2}\right)$ with $Q U f=0$. Then $U f \in \operatorname{span}(a, c)^{T}$, which can be expressed as $f=C U v(1,0)^{T}$ for some $C \neq 0$. Then the assumption $\operatorname{Pf}=M_{11} v^{*} f=0$ and the identity $V=v^{*} U v$ imply that

$$
0=M_{11} v^{*} U v(1,0)^{T}=M_{11} V(1,0)^{T}=\left(\int_{\mathbb{R}^{2}} V_{11}(y) d y\right)(1,0)^{T},
$$

where $V_{11}$ is the top left component of the matrix potential $V$. Since this argument can be reversed, we have shown that

$$
\operatorname{ker}_{Q\left(L^{2} \times L^{2}\right)}(Q U Q)=\{0\} \quad \text { if and only if } \quad \int_{\mathbb{R}^{2}} V_{11}(y) d y \neq 0 .
$$

Moreover, if $\int_{\mathbb{R}^{2}} V_{11}(y) d y=0$, then the kernel is the span of the vector $U v(1,0)^{T}$. Also note, using $V=v^{*} U v$, that

$$
V_{11}(y)=\operatorname{sign}\left(\lambda_{1}\right)|a|^{2}(y)+\operatorname{sign}\left(\lambda_{2}\right)|c|^{2}(y) .
$$


We consider first the case in which $\int_{\mathbb{R}^{2}} V_{11}(y) d y \neq 0$. In this case, $Q U Q$ is an invertible operator on $Q\left(L^{2} \times L^{2}\right)$. To see this, for any $g \in L^{2} \times L^{2}$ with $Q g=g$, define

$$
f=A g:=U g+c_{0} U v(1,0)^{T} \quad \text { with } \quad c_{0}=-\frac{\left\langle U g, v(1,0)^{T}\right\rangle}{\int_{\mathbb{R}^{2}} V_{11}(y) d y} .
$$

Note that

$$
P f=P(U g)+c_{0} P\left(U v(1,0)^{T}\right)=\frac{(a, c)^{T}}{\|(a, c)\|_{2}^{2}}\left(\left\langle U g, v(1,0)^{T}\right\rangle+c_{0} \int_{\mathbb{R}^{2}} V_{11}(y) d y\right)=0,
$$

by the definition of $c_{0}$. Therefore $Q f=f$. Also note that

$$
Q U Q f=Q U f=Q U\left(U g+c_{0} U v(1,0)^{T}\right)=g+c_{0} Q v(1,0)^{T}=g,
$$

since $Q v(1,0)^{T}=0$. Therefore, the operator $A$ is the inverse of $Q U Q$, and it is easy to see using the explicit formula that $A$ is absolutely bounded on $Q\left(L^{2} \times L^{2}\right)$.

Using resolvent identity twice, we can write

$$
D_{0}=\left(Q\left(U+v \mathcal{G}_{0} v^{*}\right) Q\right)^{-1}=A-A\left(v \mathcal{G}_{0} v^{*}\right) A+D_{0}\left(v \mathcal{G}_{0} v^{*}\right) A\left(v \mathcal{G}_{0} v^{*}\right) A
$$

The first term has already been shown to be absolutely bounded. The second term, recalling (19), is the sum of a Hilbert-Schmidt operator, $2 m v G_{0} I_{1} v^{*}$ and an operator $-i v \alpha \cdot \nabla G_{0} v^{*}$, which is bounded in absolute value by $|v| \mathcal{I}_{1}\left|v^{*}\right|$ with $\mathcal{I}_{1}$ a fractional integral operator. Recall that $\mathcal{I}_{1}: L^{2, \sigma} \rightarrow L^{2,-\sigma}$ for $\sigma>1$, see e.g. Lemma 2.3 of [34]. Therefore the second term is also absolutely bounded. Since the composition of a bounded operator with an Hilbert-Schmidt operator is Hilbert-Schmidt, and hence absolutely bounded, for the third term it suffices to prove that $\left(v \mathcal{G}_{0} v^{*}\right) A\left(v \mathcal{G}_{0} v^{*}\right)$ is Hilbert-Schmidt. This follows from the explicit formula for $A$, the inequalities

$$
\begin{aligned}
& \int_{\mathbb{R}^{2}} \frac{\langle w\rangle^{-2-}}{|x-w||w-y|} d w \lesssim 1+\log ^{-}|x-y|, \\
& \int_{\mathbb{R}^{4}}\langle x\rangle^{-2-}\left(1+\log ^{-}|x-y|\right)^{2}\langle y\rangle^{-2-} d x d y<\infty,
\end{aligned}
$$

and similar inequalities involving less singular integrands.

We now consider the case in which $\int_{\mathbb{R}^{2}} V_{11}(y) d y=0$. In this case 0 is an isolated point of the spectrum of $Q U Q$ whose essential spectrum is contained in $\{ \pm 1\}$. Let $\pi_{0}$ be the Riesz projection onto the kernel of $Q U Q$. By the calculation in the beginning of the proof, we have

$$
\pi_{0}(f)=\frac{\left\langle f, U v(1,0)^{T}\right\rangle}{\left\|(a, c)^{T}\right\|_{2}^{2}} U v(1,0)^{T} .
$$

Similarly, for $g \in Q\left(L^{2} \times L^{2}\right)$,

$$
f=A g:=U g+c_{1}\left(v(1,0)^{T}-U v(1,0)^{T}\right), \quad c_{1}=-\frac{\left\langle U g, v(1,0)^{T}\right\rangle}{\left\|(a, c)^{T}\right\|_{2}^{2}},
$$


satisfies $Q f=f$ and $\left(Q U Q+\pi_{0}\right) f=g$. By the explicit formula, $A=\left(Q U Q+\pi_{0}\right)^{-1}$ is absolutely bounded. The previous argument yields the claim by writing

$$
D_{0}=\left(Q\left(U+v \mathcal{G}_{0} v^{*}\right) Q\right)^{-1}=A-A\left(v \mathcal{G}_{0} v^{*}-\pi_{0}\right) A+D_{0}\left(v \mathcal{G}_{0} v^{*}-\pi_{0}\right) A\left(v \mathcal{G}_{0} v^{*}-\pi_{0}\right) A .
$$

Lemma 7.2. If $\left|v_{i j}(x)\right| \lesssim\langle x\rangle^{-1-}$ and $\phi \in S_{1}\left(L^{2} \times L^{2}\right)=\operatorname{Ker}(Q T Q)$ then $\phi=U v \psi$ where

$$
\psi=c_{0}(1,0)^{T}-\mathcal{G}_{0} v^{*} \phi, \quad c_{0}=\frac{\left\langle(a, c)^{T}, T \phi\right\rangle}{\left\|(a, c)^{T}\right\|_{2}^{2}} .
$$

Moreover, $\psi \in L^{\infty} \times L^{\infty}$, and it is a distributional solution of $(H-m I) \psi=0$.

Proof. Assume that $\phi \in \operatorname{Ker}(Q T Q)$. Then $Q \phi=\phi$ and $P T \phi=c_{0} v(1,0)^{T}$ by definition of the projection $P$. We have

$$
0=Q T Q \phi=(1-P) T Q \phi=T \phi-P T \phi=U \phi+v \mathcal{G}_{0} v^{*} \phi-c_{0} v(1,0)^{T} .
$$

Multiplying the above expression by $U$ on the left, we arrive at

$$
\phi=-U v \mathcal{G}_{0} v^{*} \phi+c_{0} U v(1,0)^{T}:=U v \psi
$$

where

$$
\psi=-\mathcal{G}_{0} v^{*} \phi+c_{0}(1,0)^{T} .
$$

We now prove that $(H-m I) \psi=0$. Noting that $\left(D_{m}-m I\right)(1,0)^{T}=0$, we have

$$
\begin{aligned}
& (H-m I) \psi=\left(D_{m}-m I\right) \psi+V \psi=\left(D_{m}-m I\right) \psi+v^{*} U v \psi \\
& \quad=\left(D_{m}-m I\right)\left(-\mathcal{G}_{0} v^{*} \phi+c_{0}(1,0)^{T}\right)+v^{*} \phi=-\left(D_{m}-m I\right) \mathcal{G}_{0} v^{*} \phi+v^{*} \phi .
\end{aligned}
$$

Therefore it suffices to prove that $\left(D_{m}-m I\right) \mathcal{G}_{0} v^{*} \phi=v^{*} \phi$. We compute using the definition of $\mathcal{G}_{0}$, and the identities $(-i \alpha \cdot \nabla)^{2}=-\Delta$ and $I_{2} I_{1}=0$ that

$$
\begin{aligned}
\left(D_{m}-m I\right) \mathcal{G}_{0} v^{*} \phi & =\left(-i \alpha \cdot \nabla-2 m I_{2}\right)\left(-i \alpha \cdot \nabla G_{0}+2 m G_{0} I_{1}\right) v^{*} \phi \\
= & \left(-\Delta G_{0}-2 m i \alpha \cdot \nabla G_{0} I_{1}+2 m i I_{2} \alpha \cdot \nabla G_{0}\right) v^{*} \phi \\
& =v^{*} \phi-2 m i\left(\alpha I_{1}-I_{2} \alpha\right) \cdot \nabla G_{0} v^{*} \phi=v^{*} \phi
\end{aligned}
$$

In the third equality we used $G_{0}=(-\Delta)^{-1}$, and $\left|v_{i j}(x)\right| \lesssim\langle x\rangle^{-1-}$, and the last equality follows from $\alpha_{j} I_{1}=I_{2} \alpha_{j}, j=1,2$. This proves that $(H-m I) \psi=0$.

We now prove that $\psi$ is bounded. Writing

$$
\psi=-\mathcal{G}_{0} v^{*} \phi+c_{0}(1,0)^{T}=i \alpha \cdot \nabla G_{0} v^{*} \phi-2 m G_{0} I_{1} v^{*} \phi+c_{0}(1,0)^{T},
$$

we only need to show that the first and second summands are in $L^{\infty} \times L^{\infty}$. Consider the second term. The boundedness is clear on $B(0,4)$. Then, using $M_{11} v^{*} \phi=0$ (see part i) of Remark 4.4), we can write

$$
\left[G_{0} I_{1} v^{*} \phi\right](x)=G_{0} I_{1} v^{*} \phi+\frac{1}{2 \pi} \log |x| M_{11} v^{*} \phi
$$




$$
=-\frac{1}{2 \pi} I_{1} \int_{\mathbb{R}^{2}}[\log |x-y|-\log |x|]\left(v^{*} \phi\right)(y) d y .
$$

The bound follows by using the inequality

$$
\left|\log \left(\frac{|x-y|}{|x|}\right)\right| \lesssim 1+\log (\langle y\rangle)+\log ^{-}(|x-y|), \quad|x|>4,
$$

and the bound $\left|v_{i j}(x)\right| \lesssim\langle x\rangle^{-1-}$.

To see the boundedness of the first term on the right hand side of (63), recall that

$$
\phi=-U v \mathcal{G}_{0} v^{*} \phi+c_{0} U v(1,0)^{T}=i U v \alpha \cdot \nabla G_{0} v^{*} \phi-2 m U v G_{0} I_{1} v^{*} \phi+c_{0} U v(1,0)^{T} .
$$

Note that if $f \in L^{2}\left(\mathbb{R}^{2}\right)$, for any $p \in(1,2)$ and $q \in(2, \infty), \frac{1}{q}=\frac{1}{p}-\frac{1}{2}$, we have

$$
\left\|\int \frac{1}{|x-y|}\langle y\rangle^{-1-}|f(y)| d y\right\|_{L^{q}} \lesssim\left\|\mathcal{I}_{1}\right\|_{L^{p} \rightarrow L^{q}}\left\|\langle\cdot\rangle^{-1-} f\right\|_{L^{p}} \lesssim\left\|\langle\cdot\rangle^{-1-}\right\|_{L^{q}}\|f\|_{L^{2}} \lesssim\|f\|_{L^{2}}
$$

by Lemma 6.2, the $L^{p} \rightarrow L^{q}$ boundedness of the fractional integral operator $\mathcal{I}_{1}$ in $\mathbb{R}^{2}$ and Hölder's inequality (since $\frac{1}{p}=\frac{1}{q}+\frac{1}{2}$ and $q>2$ ). This implies using the bound on $v$ that the first summand in the definition of $\phi$ is in $L^{\infty-} \times L^{\infty-}$. By $L^{\infty-}$ we mean $L^{q}$ for arbitrary large, but finite, $q$. The second summand is bounded by the argument above. Therefore $\phi \in\left(L^{\infty-} \times L^{\infty-}\right) \cap\left(L^{2} \times L^{2}\right)$. The boundedness of the first term in the definition of $\psi$ follows from this using that

$$
\begin{aligned}
& \int \frac{1}{|x-y|}\langle y\rangle^{-1-}|f(y)| d y \leq \\
&\|f\|_{L^{\infty-}}\left[\int_{|x-y|<1} \frac{1}{|x-y|^{1+}} d y\right]^{1-}+\|f\|_{L^{2}}\left[\int_{|x-y|>1}\langle y\rangle^{-2-} d y\right]^{1 / 2} \lesssim\|f\|_{L^{\infty-}}+\|f\|_{L^{2}}
\end{aligned}
$$

The following lemma provides more detailed information on $S_{1}$, however it requires more decay from the potential $V$.

Lemma 7.3. Assume that $\left|v_{i j}(x)\right| \lesssim\langle x\rangle^{-2-}$. Let $\phi=U v \psi \in S_{1}\left(L^{2} \times L^{2}\right)$. We have

$$
\psi=c_{0}(1,0)^{T}+\Gamma_{1}+\Gamma_{2},
$$

where

$$
\Gamma_{1}=-\frac{m x}{\pi\langle x\rangle^{2}} \cdot \int_{\mathbb{R}^{2}} y I_{1} v^{*}(y) \phi(y) d y-\frac{i}{2 \pi} \alpha \cdot \frac{x}{\langle x\rangle^{2}} M_{22} v^{*} \phi,
$$

and $\Gamma_{2} \in L^{p} \times L^{p}$, for any $p \in[2, \infty]$. In particular, $\psi-c_{0}(1,0)^{T} \in L^{p} \times L^{p}$ for any $2<p \leq \infty$.

Proof. Recall from Lemma 7.2 that $\psi=c_{0}(1,0)^{T}-\mathcal{G}_{0} v^{*} \phi$. Therefore, we define

$$
\Gamma_{1}+\Gamma_{2}:=-\mathcal{G}_{0} v^{*} \phi=i \alpha \cdot \nabla G_{0} v^{*} \phi-2 m G_{0} I_{1} v^{*} \phi .
$$

Below we analyze the right hand side of (66); the combination of the non- $L^{2}$ pieces gives $\Gamma_{1}$, the remaining $L^{2}$ pieces give $\Gamma_{2}$. 
We already know from Lemma 7.2 that $\psi \in L^{\infty} \times L^{\infty}$. Therefore it suffices to prove that $\Gamma_{2} \in L^{2} \times L^{2}$ on $S:=\left\{x \in \mathbb{R}^{2}:|x|>10\right\}$. We start with the second summand. We use (64) to write

$$
\begin{aligned}
&-4 \pi G_{0} I_{1} v^{*} \phi(x)=\int \log \left(\frac{|x-y|^{2}}{|x|^{2}}\right) I_{1} v^{*} \phi(y) d y \\
& \quad=\int_{A} \log \left(\frac{|x-y|^{2}}{|x|^{2}}\right) I_{1} v^{*} \phi(y) d y+\int_{B} \log \left(\frac{|x-y|^{2}}{|x|^{2}}\right) I_{1} v^{*} \phi(y) d y .
\end{aligned}
$$

Here $A:=\left\{y \in \mathbb{R}^{2}:|y|<|x| / 10\right\}, B:=\mathbb{R}^{2} \backslash A$. We note that, on the set $A,\left.|| y\right|^{2}-\left.2 x \cdot y|/| x\right|^{2}<\frac{1}{2}$, and hence

$$
\log \left(\frac{|x-y|^{2}}{|x|^{2}}\right)=\ln \left(1+\frac{|y|^{2}}{|x|^{2}}-\frac{2 x \cdot y}{|x|^{2}}\right)=-\frac{2 x \cdot y}{|x|^{2}}+O\left(\frac{\langle y\rangle^{1+}}{\langle x\rangle^{1+}}\right) .
$$

Therefore, also using that $\left|v_{i j}(y)\right| \lesssim\langle y\rangle^{-2-}$, we have

$$
\begin{array}{r}
\int_{A} \log \left(\frac{|x-y|^{2}}{|x|^{2}}\right) I_{1} v^{*} \phi d y=-\frac{2 x}{|x|^{2}} \cdot \int_{A} y I_{1} v^{*}(y) \phi(y) d y+O\left(\frac{\int_{A}\langle y\rangle^{1+}\left|v^{*}(y) \phi(y)\right| d y}{\langle x\rangle^{1+}}\right) \\
=-\frac{2 x}{|x|^{2}} \cdot \int_{A} y I_{1} v^{*}(y) \phi(y) d y+O_{L^{2}(S)}(1) .
\end{array}
$$

Note that (for $x \in S$ )

$$
\begin{aligned}
\frac{2 x}{|x|^{2}} \cdot \int_{B} y I_{1} v^{*}(y) \phi(y) d y & =O\left(\langle x\rangle^{-1}\left\|\langle y\rangle^{-1-}\right\|_{L^{2}(B)}\right)=O\left(\langle x\rangle^{-1-}\right), \\
\frac{x}{|x|^{2}}-\frac{x}{\langle x\rangle^{2}} & =O\left(\langle x\rangle^{-3}\right) .
\end{aligned}
$$

Therefore

$$
\int_{A} \log \left(\frac{|x-y|^{2}}{|x|^{2}}\right) I_{1} v^{*} \phi d y=-\frac{2 x}{\langle x\rangle^{2}} \cdot \int_{\mathbb{R}^{2}} y I_{1} v^{*}(y) \phi(y) d y+O_{L^{2}(S)}(1) .
$$

We have

$$
\left|\log \left(\frac{|x-y|^{2}}{|x|^{2}}\right)\right| \lesssim 1+\log (\langle y\rangle)+\log ^{-}(|x-y|),
$$

provided that $x \in S, y \in B$. Therefore,

$$
\begin{aligned}
&\left|\int_{B} \log \left(\frac{|x-y|^{2}}{|x|^{2}}\right) I_{1} v^{*}(y) \phi(y) d y\right| \lesssim \\
& \frac{1}{\langle x\rangle^{1+}} \int_{B}\langle y\rangle^{1+}\left(1+|y|^{0+}+|x-y|^{0-}\right)\left|v^{*}(y) \phi(y)\right| d y=O_{L^{2}(S)}(1) .
\end{aligned}
$$

This prove that

$$
-2 m G_{0} I_{1} v^{*} \phi=-\frac{m x}{\pi\langle x\rangle^{2}} \cdot \int_{\mathbb{R}^{2}} y I_{1} v^{*}(y) \phi(y) d y+O_{L^{2}(S)}(1) .
$$

Now we consider the first summand. We have

$$
i \alpha \cdot \nabla G_{0} v^{*} \phi=-\frac{i}{2 \pi} \int_{\mathbb{R}^{2}} \alpha \cdot \frac{x-y}{|x-y|^{2}} v^{*}(y) \phi(y) d y
$$




$$
=-\frac{i}{2 \pi} \int_{\mathbb{R}^{2}} \alpha \cdot\left[\frac{x-y}{|x-y|^{2}}-\frac{x}{|x|^{2}}\right] v^{*}(y) \phi(y) d y-\frac{i}{2 \pi} \alpha \cdot \frac{x}{|x|^{2}} M_{22} v^{*} \phi,
$$

since $M_{11} v^{*} \phi=0$. Therefore, the following claim and (67) finishes the proof of the lemma.

Claim:

$$
\int_{\mathbb{R}^{2}}\left|\frac{x-y}{|x-y|^{2}}-\frac{x}{|x|^{2}}\right|\left|v^{*}(y) \phi(y)\right| d y=O_{L^{2}(S)}(1) .
$$

To prove this claim first note that (for $x \in S$ )

$$
\left|\frac{x-y}{|x-y|^{2}}-\frac{x}{|x|^{2}}\right| \lesssim \begin{cases}\frac{\langle y\rangle^{0+}}{\langle x\rangle^{1+}} & y \in A, \\ \frac{\langle y\rangle^{0+}}{\langle x\rangle^{0+}|x-y|}+\frac{\langle y\rangle^{0+}}{\langle x\rangle^{1+}} & y \in B .\end{cases}
$$

The contribution of the nonsingular terms is in $L^{2}$ as above. Therefore,

$$
(\underline{68)})=\langle x\rangle^{0-} \int_{B} \frac{\langle y\rangle^{0+}}{|x-y|}\left|v^{*}(y) \phi(y)\right| d y+O_{L^{2}(S)}(1) .
$$

The integral is in $L^{p}$ for any $p>2$ because of the boundedness of the fractional integral operator $\mathcal{I}_{1}$ as in the proof of Lemma 7.2. The claim now follows from Hölder's inequality since $\langle x\rangle^{0-} \in L^{q}$ for some $q<\infty$.

Remark 7.4. i) We note that there is a threshold s-wave resonance at $\lambda=m$ for the free Dirac equation (when $V=0$ ) as the constant function $\psi=(1,0)^{T}$ solves $\left(D_{m}-m I\right) \psi=0$.

ii) One can perform a similar analysis centered near $\lambda=-m$ with $\lambda=-\sqrt{m^{2}+z^{2}}$. In this case the free equation has a threshold resonance at $\lambda=-m$ as $\psi=(0,1)^{T}$ solves $\left(D_{m}+m I\right) \psi=0$.

Lemma 7.5. Let $\left|v_{i j}(x)\right| \lesssim\langle x\rangle^{-2-}$. If $(H-m I) \psi=0$ for some $\psi=c(1,0)^{T}+\Gamma_{1}+\Gamma_{2}$ with $\Gamma_{1} \in L^{p} \times L^{p}$ for some $2<p<\infty$ and $\Gamma_{2} \in L^{2} \times L^{2}$, then $\phi:=U v \psi \in S_{1}\left(L^{2} \times L^{2}\right)$. Moreover, $\psi=c(1,0)^{T}-\mathcal{G}_{0} v^{*} \phi$ and $c=c_{0}$ as in Lemma 7.2. Furthermore, $\Gamma_{1}, \Gamma_{2} \in L^{p} \times L^{p}$ for all $p \in(2, \infty]$.

Proof. First of all, using $\phi=U v \psi$ and the assumption on $v, \psi$, we conclude that $\phi \in L^{2} \times L^{2}$ and $v^{*} \phi \in L^{1} \times L^{1}$. We have, since $(H-m I) \psi=\left(D_{m}-m I+V\right) \psi=0$,

$$
v^{*} \phi=v^{*} U v \psi=V \psi=-\left(D_{m}-m I\right) \psi .
$$

We note that $\phi \in Q\left(L^{2} \times L^{2}\right)$ if $P \phi=0$, which is equivalent to $M_{11} v^{*} \phi=\int I_{1} v^{*} \phi=0$. Using the identity above we have

$$
\left(-i \alpha \cdot \nabla+2 m I_{1}\right) v^{*} \phi=\left(D_{m}+m I\right) v^{*} \phi=-\left(D_{m}+m I\right)\left(D_{m}-m I\right) \psi=\Delta \psi .
$$

Therefore, it suffices to prove that

$$
\int \alpha \cdot \nabla\left(v^{*} \phi\right)=0, \text { and } \int \Delta \psi=0 .
$$

Both of these follow easily using $v^{*} \phi \in L^{1} \times L^{1}$, and the assumptions on $\Gamma_{1}, \Gamma_{2}$, see e.g. 35, Lemma 6.4]. Thus, $\phi \in Q\left(L^{2} \times L^{2}\right)$. 
We now claim that $\psi=c(1,0)^{T}-\mathcal{G}_{0} v^{*} \phi$. To show this, compute

$$
\begin{aligned}
\left(D_{m}-m I\right)\left(\psi+\mathcal{G}_{0} v^{*} \phi\right)=\left(D_{m}-m I\right) \psi+\left(D_{m}-m I\right) \mathcal{G}_{0} & v^{*} \phi \\
& =-V \psi+v^{*} \phi=-v^{*} \phi+v^{*} \phi=0 .
\end{aligned}
$$

In the second equality we used (62). If we apply $\left(D_{m}+m I\right)$ to this equality, we obtain

$$
-\Delta\left(\psi+\mathcal{G}_{0} v^{*} \phi\right)=0
$$

which implies that $\psi+\mathcal{G}_{0} v^{*} \phi=\left(c_{1}, c_{2}\right)^{T}$, since $\psi+\mathcal{G}_{0} v^{*} \phi \in\left(L^{2} \times L^{2}\right)+\left(L^{\infty} \times L^{\infty}\right)$ (see the proof of Lemma (7.2). Finally, since

$$
0=\left(D_{m}-m I\right)\left(\psi+\mathcal{G}_{0} v^{*} \phi\right)=\left(D_{m}-m I\right)\left(c_{1}, c_{2}\right)^{T}=-2 m\left(0, c_{2}\right)^{T},
$$

we conclude that $c_{2}=0$. Therefore, we may write $\psi=c(1,0)^{T}-\mathcal{G}_{0} v^{*} \phi$. Now, using that $Q \phi=\phi$ and the representation of $\psi$, we consider

$$
T Q \phi=\left(U+v \mathcal{G}_{0} v^{*}\right) \phi=U \phi+v\left(c(1,0)^{T}-\psi\right)=v \psi+c v(1,0)^{T}-v \psi=c v(1,0)^{T} .
$$

Therefore,

$$
Q T Q \phi=c Q v(1,0)^{T}=0, \quad \text { and } P T \phi=c P v(1,0)^{T},
$$

which implies that $\phi \in S_{1}$ and $c=c_{0}$. This finishes the proof together with Lemma 7.3.

Lemma 7.6. Assume that $\left|v_{i j}(x)\right| \lesssim\langle x\rangle^{-2-}$. Fix $\phi=U v \psi \in S_{1}\left(L^{2} \times L^{2}\right)$. Then, $\phi \in$ $S_{2}\left(L^{2} \times L^{2}\right)$ if and only if $\psi \in L^{p} \times L^{p}$ for all $p \in(2, \infty]$, that is $c_{0}=0$.

Proof. Note that $\phi \in S_{2}$ means $S_{1} T P T S_{1} \phi=0$, which holds if and only if

$$
0=\left\langle\phi, S_{1} T P T S_{1} \phi\right\rangle=\langle P T \phi, P T \phi\rangle=\|P T \phi\|_{2}^{2} .
$$

Therefore, $\phi \in S_{2}$ if and only if $c_{0}=0$. Finally note that the representation in Lemma 7.3 implies that $c_{0}=0$ if and only if $\psi \in L^{p} \times L^{p}$ for all $p \in(2, \infty]$.

Remark 7.7. i) By the representation in Lemma 7.3, if $\phi_{1}, \phi_{2} \in S_{1}$, then there is a constant c so that $\phi_{1}-c \phi_{2}$ or $\phi_{2}-c \phi_{1} \in L^{p} \times L^{p}, p \in(2, \infty]$. Therefore in $S_{2}$. This implies that the rank of $S_{1}-S_{2}$ is at most 1 .

ii) Note that $\Gamma_{1}$ in Lemma 7.3 can be written as

$$
\Gamma_{1}=w_{1} \frac{x_{1}}{\langle x\rangle^{2}}+w_{2} \frac{x_{2}}{\langle x\rangle^{2}},
$$

where the constant vectors $w_{j}$ are defined as

$$
w_{j}=-\frac{m}{\pi} \int_{\mathbb{R}^{2}} y_{j} I_{1} v^{*}(y) \phi(y) d y-\frac{i}{2 \pi} \alpha_{j} M_{22} v^{*} \phi, \quad j=1,2 .
$$

Also note that $I_{2} w_{j}=0, j=1,2$, since $I_{2} I_{1}=I_{1} M_{22}=0$ and $I_{2} \alpha_{j}=\alpha_{j} I_{1}$.

ii) Below we prove that $\phi \in S_{3}$ if and only if $\psi \in L^{2} \times L^{2}$. This and part i) imply that the rank of $S_{2}-S_{3}$ is at most 2 . 
Lemma 7.8. If $\left|v_{i j}\right| \lesssim\langle x\rangle^{-3-}$ then the operator $S_{3} v \mathcal{G}_{2} v^{*} S_{3}$ on $S_{3} L^{2}$ is invertible. Furthermore, for $f \in S_{3} L^{2}$, we have

$$
\left\langle\mathcal{G}_{2} v^{*} f, v^{*} f\right\rangle=\frac{1}{2 m}\left\langle\mathcal{G}_{0} v^{*} f, \mathcal{G}_{0} v^{*} f\right\rangle .
$$

Proof. Noting that $S_{3} v \mathcal{G}_{2} v^{*} S_{3}$ is an Hilbert Schmidt operator, it suffices to check that the kernel is empty. Given $f$ in the kernel of $S_{3} v \mathcal{G}_{2} v^{*} S_{3}$, since $S_{3} \leq S_{2} \leq S_{1} \leq Q$, we have

$$
M_{11} v^{*} f=0 \text {, and } S_{2} v \mathcal{G}_{1} v^{*} S_{2} f=0 \text {. }
$$

Using Lemma 2.2 for $\mathcal{R}_{0}(\lambda), \lambda=\sqrt{m^{2}+z^{2}}$, and using the bound on $\left|v_{i j}\right|$ we have

$$
\frac{1}{z^{2}}\left\langle\left[\mathcal{R}_{0}(\lambda)-\mathcal{G}_{0}\right] v^{*} f, v^{*} f\right\rangle=\left\langle\mathcal{G}_{2} v^{*} f, v^{*} f\right\rangle+o(1)=o(1)
$$

as $z \rightarrow 0$. Using this with $z=i w, 0<w \ll m$, we calculate the left hand side of (72)

$$
\int_{\mathbb{R}^{2}}\left\langle A(w, \xi) \widehat{v^{*} f}(\xi), \widehat{v^{*} f}(\xi)\right\rangle_{\mathbb{C}^{2}} d \xi
$$

where the Fourier multiplier $A(w, \xi)$ is given by

$$
\begin{aligned}
& A(w, \xi)=-\frac{1}{w^{2}}\left[\frac{1}{w^{2}+|\xi|^{2}}\left(\begin{array}{cc}
m+\sqrt{m^{2}-w^{2}} & \bar{\xi} \\
\xi & \sqrt{m^{2}-w^{2}}-m
\end{array}\right)-\frac{1}{|\xi|^{2}}\left(\begin{array}{cc}
2 m & \bar{\xi} \\
\xi & 0
\end{array}\right)\right] \\
& =\frac{1}{\left(w^{2}+|\xi|^{2}\right)|\xi|^{2}}\left(\begin{array}{cc}
2 m+\frac{|\xi|^{2}}{w^{2}}\left(m-\sqrt{m^{2}-w^{2}}\right) & \bar{\xi} \\
\xi & \frac{|\xi|^{2}}{w^{2}}\left(m-\sqrt{m^{2}-w^{2}}\right)
\end{array}\right) .
\end{aligned}
$$

Here $\xi=\left(\xi_{1}, \xi_{2}\right)$ is identified with $\xi_{1}+i \xi_{2}$. Note that, for $\xi \neq 0, A$ is positive definite, self-adjoint, and its eigenvalues are

$$
\lambda_{1,2}=\frac{1}{\left(w^{2}+|\xi|^{2}\right)|\xi|^{2}}\left(m+\frac{|\xi|^{2}}{w^{2}}\left(m-\sqrt{m^{2}-w^{2}}\right) \pm \sqrt{m^{2}+\xi^{2}}\right) .
$$

It is straightforward to check that $\lambda_{1,2}$ are nonincreasing functions of $w \in(0, m)$. Therefore using monotone convergence theorem after diagonalizing, we have

$$
0=\lim _{w \rightarrow 0^{+}} \int_{\mathbb{R}^{2}}\left\langle A(w, \xi) \widehat{v^{*} f}(\xi), \widehat{v^{*} f}(\xi)\right\rangle d \xi=\int_{\mathbb{R}^{2}}\left\langle A(0, \xi) \widehat{v^{*} f}(\xi), \widehat{v^{*} f}(\xi)\right\rangle_{\mathbb{C}^{2}} d \xi,
$$

where

$$
A(0, \xi)=\frac{1}{|\xi|^{4}}\left(\begin{array}{cc}
2 m+\frac{|\xi|^{2}}{2 m} & \bar{\xi} \\
\xi & \frac{|\xi|^{2}}{2 m}
\end{array}\right) .
$$

Since $A(0, \xi)$ is also positive definite and self adjoint, we conclude that $\widehat{v^{*} f}(\xi)=0$. This implies that $v^{*} f=0$ since $v^{*} f$ has $L^{1}$ entries. Recalling the definition of $v$, we obtain $\eta_{1} f_{1}=0, \eta_{2} f_{2}=0$. Also noting that $f=U v \psi=\left(\eta_{1} h_{1}, \eta_{2} h_{2}\right)$, where $h=U B \psi$, we conclude that $f=0$. Therefore $S_{3} v \mathcal{G}_{2} v^{*} S_{3}$ is invertible. 
Further, noting that

$$
A(0, \xi)=\frac{1}{2 m}\left[\frac{1}{|\xi|^{2}}\left(\begin{array}{cc}
2 m & \bar{\xi} \\
\xi & 0
\end{array}\right)\right]^{2},
$$

we obtain the identity (70) for any $f \in S_{3} L^{2}$.

Lemma 7.9. Assume that $v(x) \lesssim\langle x\rangle^{-3-}$. Fix $\phi=U v \psi \in S_{2}\left(L^{2} \times L^{2}\right)$. Then $\phi \in S_{3}\left(L^{2} \times L^{2}\right)$ if and only if $\psi \in L^{2} \times L^{2}$.

Proof. By Lemma 7.6, we have

$$
\psi=-\mathcal{G}_{0} v^{*} \phi
$$

Using this and (70), if $\phi \in S_{3}$, then we have

$$
\|\psi\|_{2}^{2}=\left\langle\mathcal{G}_{0} v^{*} \phi, \mathcal{G}_{0} v^{*} \phi\right\rangle=2 m\left\langle v \mathcal{G}_{2} v^{*} \phi, \phi\right\rangle<\infty
$$

by the decay assumption on $v$.

Now assume that $\psi \in L^{2} \times L^{2}$. Since $\frac{x_{j}}{\langle x\rangle^{2}} \notin L^{2}, j=1,2$, by Lemma 7.3 and part ii) of Remark [7.7, we have $w_{j}=0, j=1,2$, which implies that

$$
\int_{\mathbb{R}^{2}} y_{j} I_{1} v^{*}(y) \phi(y) d y=-\frac{i}{2 m} \alpha_{j} M_{22} v^{*} \phi, \quad j=1,2 .
$$

Since $\alpha_{j}^{2}=I$, this also implies that

$$
\int_{\mathbb{R}^{2}} \alpha \cdot y I_{1} v^{*}(y) \phi(y) d y=-\frac{i}{m} M_{22} v^{*} \phi
$$

We are ready to prove that $S_{2} v \mathcal{G}_{1} v^{*} S_{2} \phi=0$. Recall from (20) that

$$
\mathcal{G}_{1}=-i \alpha \cdot \nabla G_{1}+2 m G_{1} I_{1}-\frac{2}{m} M_{11}-\frac{2}{m} M_{22} \text {. }
$$

Note that the contribution of the third term is zero. We consider the contribution of the second term. We have $S_{2} v G_{1} I_{1} v^{*} S_{2}=S_{2} v W I_{1} v^{*} S_{2}$, where $W$ is the integral operator with kernel $-2 x \cdot y$. This is because $G_{1}(x, y)=|x-y|^{2}=|x|^{2}-2 x \cdot y+|y|^{2}$, and the contribution of $|x|^{2}+|y|^{2}$ is zero since $P S_{2}=S_{2} P=0$. Therefore, we have

$$
2 m S_{2} v G_{1} I_{1} v^{*} S_{2} \phi=-4 m S_{2} v(x) \int_{\mathbb{R}^{2}}(x \cdot y) I_{1} v^{*}(y) \phi(y) d y=2 i S_{2} v(x)(\alpha \cdot x) M_{22} v^{*} \phi .
$$

In the second equality we used (73). The contribution of the first term is

$$
\begin{aligned}
-i S_{2} v \alpha \cdot \nabla G_{1} v^{*} \phi=-2 i S_{2} v & (x) \int_{\mathbb{R}^{2}} \alpha \cdot(x-y) v^{*}(y) \phi(y) d y \\
=-2 i S_{2} v(x)(\alpha \cdot x) & \int_{\mathbb{R}^{2}} v^{*}(y) \phi(y) d y+2 i S_{2} v(x) \int_{\mathbb{R}^{2}} \alpha \cdot y v^{*}(y) \phi(y) d y \\
& =-2 i S_{2} v(x)(\alpha \cdot x) M_{22} v^{*} \phi+2 i S_{2} v(x) I_{2} \int_{\mathbb{R}^{2}} \alpha \cdot y v^{*}(y) \phi(y) d y .
\end{aligned}
$$


In the last equality we used $P S_{2}=S_{2} P=0$. Therefore, the sum of the contributions of the first two terms in the definition of $\mathcal{G}_{1}$ is equal to (using (74))

$$
2 i S_{2} v(x) I_{2} \int_{\mathbb{R}^{2}} \alpha \cdot y v^{*}(y) \phi(y) d y=2 i S_{2} v(x) \int_{\mathbb{R}^{2}} \alpha \cdot y I_{1} v^{*}(y) \phi(y) d y=\frac{2}{m} S_{2} v(x) M_{22} v^{*} \phi,
$$

which cancels the contribution of the last term in the definition of $\mathcal{G}_{1}$. This finishes the proof of the lemma.

Lemma 7.10. Assume that $v(x) \lesssim\langle x\rangle^{-3-}$. The operator

$$
P_{m}:=\frac{1}{2 m} \mathcal{G}_{0} v S_{3}\left[S_{3} v \mathcal{G}_{2} v^{*} S_{3}\right]^{-1} S_{3} v^{*} \mathcal{G}_{0}
$$

is the finite rank orthogonal projection onto the $m$ energy eigenspace of $H=D_{m}+V$.

Proof. Let $\left\{\phi_{j}\right\}_{j=1}^{N}$ be an orthonormal basis for the $S_{3} L^{2}$, the range of $S_{3}$. By the characterization in Lemma 7.2 and Remark 4.4, the eigenspace is finite dimensional. Then, by the lemmas above, we have

$$
\phi_{j}=U v \psi_{j}, \quad \psi_{j}=-\mathcal{G}_{0} v^{*} \phi_{j}, \quad 1 \leq j \leq N,
$$

where $\psi_{j} \in L^{2} \times L^{2}, j=1,2, \ldots, N$, are eigenvectors. Since $\left\{\phi_{j}\right\}_{j=1}^{N}$ is linearly independent, we have that $\left\{\psi_{j}\right\}_{j=1}^{N}$ is linearly independent, and hence it is a basis for $m$ energy eigenspace. Using the orthonormal basis for $S_{3} L^{2}$, we have that for any $f \in L^{2} \times L^{2}, S_{3} f=\sum_{j=1}^{N}\left\langle f, \phi_{j}\right\rangle \phi_{j}$. Therefore, we have

$$
S_{3} v^{*} \mathcal{G}_{0} f=\sum_{j=1}^{N}\left\langle f, \mathcal{G}_{0} v^{*} \phi_{j}\right\rangle \phi_{j}=-\sum_{j=1}^{N}\left\langle f, \psi_{j}\right\rangle \phi_{j} .
$$

This implies that the range of $P_{m}$ is contained in the span of $\left\{\psi_{j}\right\}_{j=1}^{N}$, since $P_{m}$ is self-adjoint.

We claim that, for each $i_{0}, j_{0} \in\{1,2, \ldots, N\}$,

$$
\left\langle\psi_{i_{0}}, P_{m} \psi_{j_{0}}\right\rangle=\left\langle\psi_{i_{0}}, \psi_{j_{0}}\right\rangle \text {. }
$$

This implies that the range of $P_{m}$ is equal to the span of $\left\{\psi_{j}\right\}_{j=1}^{N}$ and that $P_{m}$ is the identity operator on the range of $P_{m}$. Since $P_{m}$ is self-adjoint, the assertion of the lemma holds.

Let $\mathcal{A}:=S_{3} v \mathcal{G}_{2} v^{*} S_{3}$. Let $A=\left\{A_{i j}\right\}_{i, j=1}^{N}, B=\left\{B_{i j}\right\}_{i, j=1}^{N}$ be the matrix representations of $\mathcal{A}$ and $\mathcal{A}^{-1}$ with respect to the orthonormal basis $\left\{\phi_{j}\right\}_{j=1}^{N}$ of $S_{3}$. Using (70) and polarization,

$$
\begin{aligned}
& A_{i j}=\left\langle\phi_{j}, S_{3} v \mathcal{G}_{2} v^{*} S_{3} \phi_{i}\right\rangle=\frac{1}{2 m}\left\langle\mathcal{G}_{0} v^{*} \phi_{j}, \mathcal{G}_{0} v^{*} \phi_{i}\right\rangle=\frac{1}{2 m}\left\langle\psi_{j}, \psi_{i}\right\rangle, \\
& B_{i j}=A_{i j}^{-1}=\left\langle\phi_{j}, \mathcal{A}^{-1} \phi_{i}\right\rangle .
\end{aligned}
$$

Using this and (76), we have

$$
\left\langle\psi_{i_{0}}, P_{m} \psi_{j_{0}}\right\rangle=\frac{1}{2 m}\left\langle S_{3} v^{*} \mathcal{G}_{0} \psi_{i_{0}}, \mathcal{A}^{-1} S_{3} v^{*} \mathcal{G}_{0} \psi_{j_{0}}\right\rangle
$$




$$
\begin{array}{r}
=\frac{1}{2 m}\left\langle\sum_{i=1}^{N}\left\langle\psi_{i_{0}}, \psi_{i}\right\rangle \phi_{i}, \mathcal{A}^{-1} \sum_{j=1}^{N}\left\langle\psi_{j_{0}}, \psi_{j}\right\rangle \phi_{j}\right\rangle=\frac{1}{2 m} \sum_{i, j=1}^{N}\left\langle\psi_{i_{0}}, \psi_{i}\right\rangle\left\langle\psi_{j}, \psi_{j_{0}}\right\rangle\left\langle\phi_{i}, \mathcal{A}^{-1} \phi_{j}\right\rangle \\
=2 m \sum_{i, j=1}^{N} A_{i, i_{0}} B_{j, i} A_{j_{0}, j}=2 m A_{j_{0}, i_{0}}=\left\langle\psi_{i_{0}}, \psi_{j_{0}}\right\rangle .
\end{array}
$$

This finishes the proof of the claim and the lemma.

\section{ACKNOWLEDGEMEnTS}

The authors would like to thank Nabile Boussaid and the anonymous referee for many helpful suggestions. In particular, suggesting relevant work on the spectrum of Dirac operators which strengthened our results.

\section{REFERENCES}

[1] Abramowitz, M. and I. A. Stegun. Handbook of mathematical functions with formulas, graphs, and mathematical tables. National Bureau of Standards Applied Mathematics Series, 55. For sale by the Superintendent of Documents, U.S. Government Printing Office, Washington, D.C. 1964

[2] Agmon, S. Spectral properties of Schrödinger operators and scattering theory. Ann. Scuola Norm. Sup. Pisa Cl. Sci. (4) 2 (1975), no. 2, 151-218.

[3] Aharonov, Y., and Casher, A. Ground state of a spin-1/2 charged particle in a two-dimensional magnetic field. Phys. Rev. A (3) 19 (1979), no. 6, 2461-2462.

[4] Arai, M., and Yamada, O. Essential selfadjointness and invariance of the essential spectrum for Dirac operators. Publ. Res. Inst. Math. Sci. 18 (1982), no. 3, 973-985.

[5] Beceanu, M. Decay estimates for the wave equation in two dimensions. J. Differential Equations 260 (2016), no. $6,5378-5420$.

[6] Bejenaru, I., and Herr, S. The cubic Dirac equation: small initial data in $H^{1 / 2}\left(\mathbb{R}^{2}\right)$. Commun. Math. Phys. 343 (2016), 515-562.

[7] Bennett, C., and Sharpley, R. Interpolation of operators. Pure and Applied Mathematics, 129. Academic Press, Inc., Boston, MA, 1988.

[8] A. Berthier and V. Georgescu, On the point spectrum of Dirac operators. J. Funct. Anal. 71 (1987), no. 2, 309-338.

[9] Bollé, D., Danneels, C., and F. Gesztesy. Threshold scattering in two dimensions. Ann. Inst. H. Poincaré Phys. Théor. 48 (1988), no. 2, 175-204.

[10] Boussaid, N. Stable directions for small nonlinear Dirac standing waves. Comm. Math. Phys. 268 (2006), no. 3, 757-817.

[11] Boussaid, N., D'Ancona P., and Fanelli, L. Virial identiy and weak dispersion for the magnetic Dirac equation. J. Math. Pures Appl., 95:137-150, 2011.

[12] Boussaid, N., and Golenia, S. Limiting absorption principle for some long range perturbations of Dirac systems at threshold energies. Comm. Math. Phys. 299 (2010), no. 3, 677-708.

[13] Cacciafesta, F. Virial identity and dispersive estimates for the n-dimensional Dirac equation, J. Math. Sci. Univ. Tokyo 18 (2011), 1-23. 
[14] Cacciafesta, F., and Seré, E. Local smoothing estimates for the massless Dirac equation in 2 and 3 dimensions. To appear in J. Func. Anal. http://arxiv.org/abs/1503.00945

[15] P. A. Cojuhari, On the finiteness of the discrete spectrum of the Dirac operator. Rep. Math. Phys. 57 (2006), no. 3, 333-341.

[16] P. A. Cojuhari, Finiteness of eigenvalues of the perturbed Dirac operator. Operator theory, analysis and mathematical physics, 1-7, Oper. Theory Adv. Appl., 174, Birkhauser, Basel, 2007.

[17] Comech, A., Phan, T., and Stefanov, A. Asymptotic stability of solitary waves in generalized Gross-Neveu model. To appear in Ann. Inst. H. Poincaré Anal. Non Linéaire .

[18] D'Ancona, P., and Fanelli, L. $L^{p}$-boundedness of the wave operator for the one dimensional Schrödinger operator. Comm. Math. Phys. 268 (2006), no. 2, 415-438.

[19] D'Ancona, P., and Fanelli, L. Decay estimates for the wave and Dirac equations with a magnetic potential. Comm. Pure Appl. Math. 60 (2007), no. 3, 357-392.

[20] Ding, Y., and Lu, S. Homogeneous fractional integrals on Hardy spaces. Tohoku Math. J. (2) 52 (2000), no. 1, 153-162.

[21] Erdoğan, M. B., Goldberg, M, and Schlag, W. Strichartz and smoothing estimates for Schrödinger operators with almost critical magnetic potentials in three and higher dimensions. Forum Math. 21 (2009), 687-722.

[22] Erdoğan, M. B., and Green, W. R. Dispersive estimates for the Schrodinger equation for $C^{\frac{n-3}{2}}$ potentials in odd dimensions. Int. Math. Res. Notices 2010:13, 2532-2565.

[23] Erdoğan, M. B., and Green, W. R. Dispersive estimates for Schrödinger operators in dimension two with obstructions at zero energy. Trans. Amer. Math. Soc. 365 (2013), 6403-6440.

[24] Erdoğan, M. B., and Green, W. R. A weighted dispersive estimate for Schrödinger operators in dimension two. Commun. Math. Phys. 319 (2013), 791-811.

[25] Erdoğan, M. B., and Green, W. R. Dispersive estimates for matrix Schrödinger operators in dimension two. Discrete Contin. Dyn. Syst. 33 (2013), no. 10, 4473-4495.

[26] Erdoğan, M. B., Green, W. R. and Toprak, E. Dispersive estimates for Dirac operators in dimension three with obstructions at threshold energies. Submitted 2016, 36 pages. arXiv:1609.05164

[27] Escobedo, M., and Vega, L. A semilinear Dirac equation in $H^{s}\left(\mathbb{R}^{3}\right)$ for $s>1$. SIAM J. Math. Anal. 28 (1997), no. 2, 338-362.

[28] Fefferman, C., and Stein, E. M. $H^{p}$ spaces of several variables. Acta Math. 129 (1972), no. 3-4, $137-193$.

[29] Georgescu, V., and Mantoiu, M. On the spectral theory of singular Dirac type Hamiltonians. J. Operator Theory 46 (2001), no. 2, 289-321.

[30] Ginibre, J., and Velo, G. Generalized Strichartz inequalities for the wave equation, J. Funct. Anal. 133 (1995) 50-68.

[31] Green, W. Time decay estimates for the wave equation with potential in dimension two. J. Differential Equations 257 (2014), no. 3, 868-919.

[32] Hanks, R. Interpolation by the real method between BMO, $L^{\alpha}(0<\alpha<\infty)$ and $H^{\alpha}(0<\alpha<\infty)$. Indiana Univ. Math. J. 26 (1977), no. 4, 679-689.

[33] Hunziker, W., Sigal, I.M., Soffer, A. Minimal escape velocities. Comm. Partial Differ. Eqs. 24 (11-12), 22792295.

[34] Jensen, A. Spectral properties of Schrödinger operators and time-decay of the wave functions results in $L^{2}\left(R^{m}\right), m \geq 5$. Duke Math. J. 47 (1980), no. 1, 57-80.

[35] Jensen, A., and G. Nenciu. A unified approach to resolvent expansions at thresholds. Rev. Mat. Phys. vol. 13, no. 6 (2001), $717-754$. 
[36] E. Kopylova Dispersion estimates for 2D Dirac equation. Asymptot. Anal. 84 (2013), no. 1-2, 35-46.

[37] Kurbenin, O. I. The discrete spectra of the Dirac and Pauli operators. Topics in Mathematical Physics, Vol. 3, Spectral Theory, p.43-52, M.S. Birman ed., Consultants Bureau, 1969.

[38] Murata, M. Asymptotic expansions in time for solutions of Schrödinger-type equations J. Funct. Anal. 49 (1) (1982), 10-56.

[39] Reed, M., and B. Simon. Methods of Modern Mathematical Physics I: Functional Analysis, IV: Analysis of Operators, Academic Press, New York, NY, 1972.

[40] Roze, S.N., On the character of the spectrum of the Dirac operator, Theor. Math. Phys. 2 (1970), 377-382.

[41] Schlag, W. Dispersive estimates for Schrödinger operators in dimension two. Comm. Math. Physi. 257 (2005), no. $1,87-117$.

[42] Thaller, B. The Dirac equation. Texts and Monographs in Physics. Springer-Verlag, Berlin, 1992.

[43] M. Thompson, The absence of embedded eigenvalues in the continuous spectrum for perturbed Dirac operators. Boll. Un. Mat. Ital. A (5) 13 (1976), no. 3, 576-585.

[44] Toprak, E. A weighted estimate for two dimensional Schrödinger, Matrix Schrödinger and wave equations with resonance of the first kind at zero. preprint 2015, to appear in J. Spectr. Theory.

[45] Vogelsang, V. Absence of embedded eigenvalues of the Dirac equation for long range potentials. Analysis 7 (1987), no. 3-4, 259-274.

[46] Yamada, O. A remark on the limiting absorption method for Dirac operators. Proc. Japan Acad. Ser. A Math. Sci. 69 (1993), no. 7, 243-246.

Department of Mathematics, University of Illinois, Urbana, IL 61801, U.S.A.

E-mail address: berdogan@math.uiuc.edu

Department of Mathematics, Rose-Hulman Institute of Technology, Terre Haute, IN 47803, U.S.A.

E-mail address: green@rose-hulman.edu 